\title{
Transition state spectroscopy of the excited electronic states of $\mathrm{Li}-\mathrm{HF}$
}

\author{
Alfredo Aguado and Miguel Paniagua ${ }^{\text {a) }}$ \\ Departamento de Química Física, Facultad de Ciencias C-XIV, Universidad Autónoma de Madrid, \\ 28049 Madrid, Spain \\ Cristina Sanz and Octavio Roncero ${ }^{\text {b) }}$ \\ Instituto de Matemáticas y Física Fundamental, C.S.I.C., Serrano 123, 28006 Madrid, Spain
}

(Received 21 July 2003; accepted 22 August 2003)

\begin{abstract}
In this work the $\operatorname{LiHF}\left(A, B, B^{\prime} \leftarrow X\right)$ electronic spectrum is simulated and compared with the experimental one obtained by Hudson et al. [J. Chem. Phys. 113, 9897 (2000)]. High level ab initio calculations of three ${ }^{2} A^{\prime}$ and one ${ }^{2} A^{\prime \prime}$ electronic states have been performed using a new atomic basis set and for a large number of nuclear configurations (about 6000). Four analytic global potential energy surfaces have been fitted. The spectrum involved very excited rovibrational states, close to the first dissociation limit, at high total angular momentum. Two different methods have been used, one based on bound state and the second one on wave packet calculations. Different alternatives have been used to simulate the relatively high temperatures involved. The agreement obtained with the experimental spectrum is very good allowing a very simple assignment of the peaks. They are due to bending progressions on the three excited electronic states. A simple model is used in which only rotational degrees of freedom are included, which simulates the spectrum in excellent agreement with the experimental one, providing a nice physical interpretation. Moreover, the remaining theoretical/experimental discrepancies have been attributed to nonadiabatic effects through the extension of this model to a diabatic representation of excited coupled electronic states. (C) 2003 American Institute of Physics. [DOI: 10.1063/1.1618223]
\end{abstract}

\section{INTRODUCTION}

Reactivity involves a reordering of the electronic structure when evolving from reactant to products. Even in the case of a single Born-Oppenheimer state, the ground electronic state can be viewed as resulting from the diagonalization of a Hamiltonian matrix represented in a crude basis of the "diabatic" electronic states characterized by the asymptotic states of the reactant and product fragments they correlate to. Thus, the barriers appearing in the minimum energy path between reactants and products can be interpreted as a result of a crossing among different electronic states. Such singular region, or transition state, acts as a bottleneck for the reaction, determining the reaction mechanism and many of the properties of the measured magnitudes. However, because in this region the electronic structure suffers a sudden change and nonadiabatic couplings are usually large, it is important to include several states and nonadiabatic effects to understand and simulate the reaction mechanism at electronic level.

For these kind of studies, systems composed by an alkali or alkaline earth atom (M) with RX halides (with $\mathrm{X}$ being a halogen atom and $\mathrm{R}=\mathrm{H}, \mathrm{CH}_{3}$, etc.) can be considered as prototypes: the ground electronic state is the result of a crossing between one/several covalent states and one/several ionic states correlating with the $\mathrm{M}^{*}+\mathrm{XR}$ reactants and with the $\left(\mathrm{M}^{+} \mathrm{X}^{-}\right)^{*}+\mathrm{R}$ products, respectively. Therefore the

\footnotetext{
a) Author to whom all correspondence should be addressed. Electronic mail: miguel.paniagua@uam.es

${ }^{b)}$ Electronic mail: ORoncero@imaff.cfmac.csic.es
}

$\mathrm{M}^{*}+\mathrm{XR} \rightarrow\left(\mathrm{M}^{+} \mathrm{X}^{-}\right)^{*}+\mathrm{R}$ reactions are envisaged as the transfer of an electron of the alkali atom to the halogen atom ${ }^{1,2}$ ("harpoon" mechanism) at precise configurations, where the curve crossing occurs and it is interesting to determine how nonadiabatic terms affect the reaction dynamics. After the pioneer work of Taylor and Datz, ${ }^{3}$ a large amount of experimental cross beams studies on these systems was done in 1960s, ${ }^{4}$ the so-called "alkali age." Thus, there is a wealth of experimental information about the influence of the excitation (electronic, ${ }^{5,6}$ vibrational, $^{7-13}$ and rotational ${ }^{14-17}$ ) of the reactants on these systems under collision conditions. In contrast, there are only a few studies devoted to simulate nonadiabatic effects.

Another important feature of these systems is the presence of van der Waals wells in the entrance channel due to the strong dipole electric fields of the reactants. Thus, it is possible to form the complex among the reactants and promote the system to excited electronic states, and monitor the appearance of reactant and/or products. After the first experiments by Soep and co-workers on $\mathrm{Ca}-\mathrm{HX}$ systems, ${ }^{18-22}$ there have been several other studies on related systems by the groups of Polanyi, ${ }^{23-27}$ González-Ureña, ${ }^{28-30}$ etc. More recently also time resolved experiments ${ }^{31,32}$ have opened the possibility of following the evolution of the reaction in real time and also to "control" the course of the reaction by properly designed excitation pulses. These kind of results provide detailed information about the excited electronic states and about the nonadiabatic couplings governing the transitions between the different electronic states correlating to different final states of products. 
Among all these systems LiHF can be considered as a benchmark model system because of its relative simplicity: it has 13 electrons, which allows relatively accurate $a b$ initio calculations, only one single electronic state of $\mathrm{LiF}$ products, is one of the lightest systems with three different atoms, and still presents a very rich dynamics. Aside from the experimental problems related to its study, there are several works on the $\mathrm{Li}+\mathrm{HF}$ collisions, ${ }^{33-38}$ and the $\operatorname{LiHF}\left(A, B, B^{\prime} \leftarrow X\right)$ electronic absorption spectrum has recently been measured by Polanyi and co-workers. ${ }^{26}$ On the theoretical side, there is an increasing number of $a b$ initio studies to characterize the potential energy surfaces (PESs) on the ground ${ }^{39-43}$ and more recently also on the excited ${ }^{44,45,42}$ electronic states, and many theoretical studies to simulate the collision, using quantum $^{39,40,46-49}$ as well as classical ${ }^{50,37}$ methods on the ground electronic state. Also some theoretical studies have been devoted to the spectroscopic study of the transition state by infrared excitation on the ground electronic state..$^{51-53}$ However, there are only a few and approximate dynamical studies about the nonadiabatic dynamics of this system, especially devoted to the electronic predissociation decay from the excited electronic states. ${ }^{44,54,42}$

The aim of this work is to fully characterize the electronic absorption spectrum of $\operatorname{LiHF}\left(A, B, B^{\prime} \leftarrow X\right)$ recently measured by Polanyi and co-workers. ${ }^{26}$ Nonadiabatic dynamics will be left for a forthcoming work. An important accuracy improvement on the PESs is obtained with respect to a previous study. ${ }^{45}$ In Sec. II, the ab initio electronic structure calculations will be described and in Sec. III the analytic fits generated to the global PESs will be compared with previous ones, when available. In Sec. IV the methods used to simulate the absorption spectra are briefly described. The results are compared with the experimental spectrum of Polanyi and co-workers ${ }^{26}$ and a complete assignment is discussed. Finally, some conclusions are established in Sec. V.

\section{AB INITIO ELECTRONIC STRUCTURE CALCULATIONS}

\section{A. Electronic basis set, atomic and diatomic energies}

The interest of this work focuses on the ground and first excited states of LiHF, correlating to $\operatorname{Li}\left({ }^{2} S\right)+\mathrm{HF}\left(X^{1} \Sigma^{+}\right)$ and $\operatorname{Li}\left({ }^{2} P\right)+\operatorname{HF}\left(X^{1} \Sigma^{+}\right)$dissociation limits. It is also important to properly describe the $\mathrm{Li}^{+}+\mathrm{HF}^{-}$and $\mathrm{Li}^{+}+\mathrm{H}^{-} \mathrm{F}$ ionic configurations since they are responsible for the curve crossing leading to the LiF products in the ground BornOppenheimer electronic state. The saddle point appears as a result of the crossing between the $\mathrm{Li}^{+}+\mathrm{HF}^{-}$ionic state and a covalent configuration correlating to $\operatorname{Li}\left({ }^{2} S\right)+\operatorname{HF}\left(X^{1} \Sigma^{+}\right)$ (see Fig. 1). In addition, following the arguments of Zeiri and Shapiro in a diatomics-in-molecules model for this system, ${ }^{55,56}$ the $\mathrm{Li}^{+}+\mathrm{HF}^{-}$state is strongly stabilized by a resonance phenomena with another ionic configuration, $\mathrm{Li}^{+}$ $+\mathrm{H}^{-} \mathrm{F}$, at very precise angular configurations, which explains the bent character of the saddle point. This crossing takes place at a quite short distance, where the attractive Coulombic interaction between ions of opposite sign compensates for the high asymptotic energies of the isolated ions, $\mathrm{Li}^{+}$and $\mathrm{HF}^{-}$. When the two ions approach each other, the

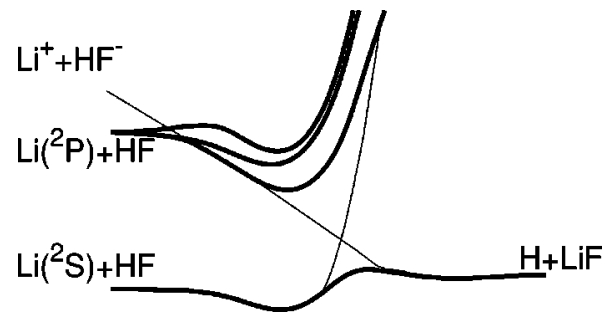

FIG. 1. Minimum energy path diagram for the $X, A, B^{\prime}$, and $B$ electronic states of Li-HF (ordered in energy).

energy decreases until it cuts, not only with the ground $\mathrm{Li}\left({ }^{2} S\right)+\mathrm{HF}\left(X^{1} \Sigma^{+}\right)$covalent configuration, but also with the excited $\operatorname{Li}\left({ }^{2} P\right)+\operatorname{HF}\left(X^{1} \Sigma^{+}\right)$ones. These crossings take place at much longer distances and therefore the use of very good atomic basis sets to properly describe the polarization of the electronic clouds is very important. Just as an example, the crossings of the energy curve of the ionic $\mathrm{F}^{-} \mathrm{Li}^{+}$ state with those of the covalent configurations correlating to $\mathrm{F}\left({ }^{2} P\right)+\mathrm{Li}\left({ }^{2} S\right)$ and $\mathrm{F}\left({ }^{2} P\right)+\mathrm{Li}\left({ }^{2} P\right)$ are shown in Fig. 2, which take place at $\approx 13$ and 150 a.u., respectively.

As a result, special attention was paid to determining the atomic basis set. The electronic basis set used in the ab initio

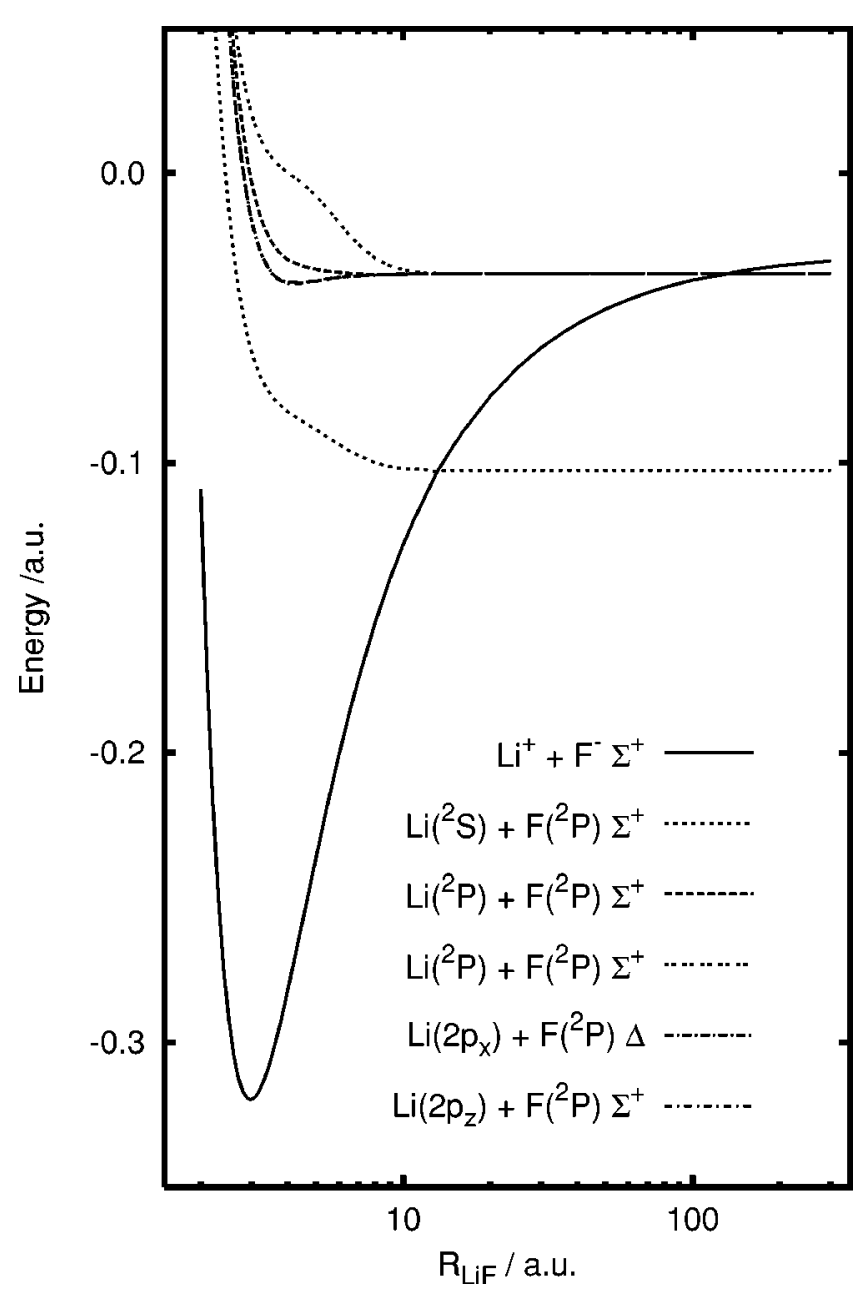

FIG. 2. Energy levels of the $\operatorname{LiF}\left({ }^{1} \Sigma\right)$ states for the first electronic states, correlating to the $\left.\mathrm{F}\left({ }^{2} P\right)+\mathrm{Li}\left({ }^{2} S\right), \mathrm{F}^{2} P\right)+\mathrm{Li}\left({ }^{2} P\right)$ and $\mathrm{F}^{-}\left({ }^{1} S\right)+\mathrm{Li}^{+}\left({ }^{1} S\right)$ asymptotes, with the internuclear distance in logarithmic scale. 
TABLE I. Calculated and experimental excitation energies, ionization potential (IP), and electron affinities (EA) of the atoms. The experimental values were taken from Ref. 82.

\begin{tabular}{ccrrr}
\hline \hline Atom & & $\begin{array}{c}\text { MRCI }+Q \\
\left(\mathrm{~cm}^{-1}\right)\end{array}$ & $\begin{array}{c}\text { Expt. } \\
\left(\mathrm{cm}^{-1}\right)\end{array}$ & \multicolumn{1}{c}{$\begin{array}{c}\text { Error } \\
\left(\mathrm{cm}^{-1}\right)\end{array}$} \\
\hline $\mathrm{Li}$ & & & & \\
& $\ldots 2 s^{2} S \rightarrow \ldots 2 p^{2} P$ & 14950.3 & 14903.5 & +46.8 \\
& $\ldots 2 s^{2} S \rightarrow \ldots 3 s^{2} S$ & 27176.0 & 27205.9 & -29.9 \\
& $\ldots 2 s^{2} S \rightarrow \ldots 3 p^{2} P$ & 30911.2 & 30924.1 & -12.9 \\
& $\ldots 2 s^{2} S \rightarrow \ldots 3 d^{2} D$ & 31251.6 & 31282.2 & -30.6 \\
& $\mathrm{IP}$ & 43442.6 & 43486.2 & -43.6 \\
& $\mathrm{AE}$ & 4957.1 & 4982.1 & -25.0 \\
$\mathrm{~F}$ & $\mathrm{IP}$ & 139098.3 & 140525.9 & -1427.6 \\
& $\mathrm{EA}$ & 27277.7 & 27430.9 & -153.2 \\
& & & & \\
$\mathrm{H}$ & $1 s^{12} S \rightarrow 2 s^{12} S$ & 82268.5 & 82300.8 & -32.3 \\
& $1 s^{12} S \rightarrow 2 p^{12} P$ & 82292.7 & 82300.8 & -8.1 \\
& $\mathrm{AE}$ & 5972.5 & 6079.0 & 106.5 \\
\hline \hline & & & &
\end{tabular}

calculations consisted of an extended basis for the lithium atom of size $(15 s, 11 p, 4 d, 1 f) /[14 s, 10 p, 3 d, 1 f]$, and the aug-cc-pVTZ basis set of Dunning et al. ${ }^{57,58}$ for the hydrogen and fluorine atoms, augmented by several diffuse functions whose exponents were optimized to accurately reproduce excited energies of the lithium atom, as well as ionization potential (IP) and electron affinities (EA) of the three atoms. The lithium basis set was obtained by using as a starting point a combination of two different basis sets. For the inner electrons, part of the basis set used by Yarkony to study $\mathrm{LiHCl}^{59}$ was used, and for the outer ones, the diffuse functions of the basis set of Boutalib and Gadéa. ${ }^{60}$ This basis was later optimized to reproduce the excited states of lithium. The atomic spectrum for $\mathrm{Li}$ is reproduced in Table I, with a residual error for the excited Rydberg states, IP and EA lower than $\pm 50 \mathrm{~cm}^{-1}$. For the fluorine and hydrogen atoms the most important quantities are the EA, shown also in Table I, which in the present calculations have residual errors lower than $150 \mathrm{~cm}^{-1}$.

The $a b$ initio spectroscopic magnitudes for different electronic states of the $\mathrm{LiF}, \mathrm{HF}$, and $\mathrm{LiH}$ diatomic fragments show a rather good agreement with the experimental data, especially those magnitudes related to the first excited electronic states more directly involved in the triatomic system under study.

\section{B. Triatomic ab initio calculations}

For the LiHF system the three lowest states of ${ }^{2} A^{\prime}$ symmetry $\left(X^{2} A^{\prime}, A^{2} A^{\prime}\right.$ and $\left.B^{2} A^{\prime}\right)$, as well as the lowest state of ${ }^{2} A^{\prime \prime}$ symmetry $\left(B^{\prime 2} A^{\prime \prime}\right)$ have been calculated. The $X^{2} A^{\prime}$ ground state correlates adiabatically with the reactants' and products' ground states:

$$
\begin{aligned}
\operatorname{Li}\left({ }^{2} S\right)+\mathrm{HF}\left(X^{1} \Sigma^{+}\right) & \rightarrow \operatorname{LiFH}\left(X^{2} A^{\prime}\right) \\
& \rightarrow\left\{\begin{array}{l}
\operatorname{LiF}\left(X^{1} \Sigma^{+}\right)+\mathrm{H}\left({ }^{2} S\right) \\
\operatorname{LiH}\left(X^{1} \Sigma^{+}\right)+\mathrm{F}\left({ }^{2} P\right)
\end{array}\right.
\end{aligned}
$$

The excited states, nevertheless, correlate adiabatically with the same reactants limit corresponding to the lithium excited state $\operatorname{Li}\left({ }^{2} P\right)+\operatorname{HF}\left(X^{1} \Sigma^{+}\right)$if $R_{\mathrm{HF}}<3.5$ a.u. or with several HF excited states $\operatorname{Li}\left({ }^{2} S\right)+\operatorname{HF}\left(C^{1} \Pi\right)$ or $\operatorname{Li}\left({ }^{2} S\right)+\operatorname{HF}\left(b^{3} \Pi\right)$ if $R_{\mathrm{HF}}>3.5$ a.u. (see Fig. 2 of Ref. 44 ):

$$
\begin{aligned}
& \mathrm{Li}\left({ }^{2} P\right)+\mathrm{HF}\left(X^{1} \Sigma^{+}\right) \quad \text { if } R_{\mathrm{HF}}<3.5 \text { a.u. } \\
& \mathrm{Li}\left({ }^{2} S\right)+\mathrm{HF}\left(b^{3} \Pi\right) \quad \text { if } R_{\mathrm{HF}}>3.5 \text { a.u. } \\
& \rightarrow \operatorname{LiFH}\left(A^{2} A^{\prime}\right) \rightarrow\left\{\begin{array}{l}
\operatorname{LiF}\left(b^{3} \Pi\right)+\mathrm{H}\left({ }^{2} S\right) \\
\operatorname{LiH}\left(X^{1} \Sigma^{+}\right)+\mathrm{F}\left({ }^{2} P\right)
\end{array},\right. \\
& \mathrm{Li}\left({ }^{2} P\right)+\mathrm{HF}\left(X^{1} \Sigma^{+}\right) \text {if } R_{\mathrm{HF}}<3.5 \text { a.u. } \\
& \mathrm{Li}\left({ }^{2} S\right)+\mathrm{HF}\left(C^{1} \Pi\right) \quad \text { if } R_{\mathrm{HF}}>3.5 \text { a.u. } \\
& \rightarrow \operatorname{LiFH}\left(B^{2} A^{\prime}\right) \rightarrow\left\{\begin{array}{l}
\operatorname{LiF}\left(A^{1} \Pi\right)+\mathrm{H}\left({ }^{2} S\right) \\
\operatorname{LiH}\left(a^{3} \Sigma^{+}\right)+\mathrm{F}\left({ }^{2} P\right)
\end{array},\right.
\end{aligned}
$$

and

$$
\begin{aligned}
& \mathrm{Li}\left({ }^{2} P\right)+\mathrm{HF}\left(X^{1} \Sigma^{+}\right) \quad \text { if } R_{\mathrm{HF}}<3.5 \text { a.u. } \\
& \mathrm{Li}\left({ }^{2} S\right)+\mathrm{HF}\left(b^{3} \Pi\right) \quad \text { if } R_{\mathrm{HF}}>3.5 \text { a.u. } \\
& \rightarrow \operatorname{LiFH}\left(B^{\prime 2} A^{\prime \prime}\right) \rightarrow\left\{\begin{array}{l}
\operatorname{LiF}\left(b^{3} \Pi\right)+\mathrm{H}\left({ }^{2} S\right) \\
\operatorname{LiH}\left(X^{1} \Sigma^{+}\right)+\mathrm{F}\left({ }^{2} P\right)
\end{array} .\right.
\end{aligned}
$$

On the other hand, note that because the excited states share several dissociation limits, there are conical intersections corresponding to the $C_{2 v} \rightarrow C_{s}$ displacement, which makes the $\mathrm{LiHF}^{2} \Pi$ correlate adiabatically with the LiHF ${ }^{2} A^{\prime}$ and ${ }^{2} A^{\prime \prime}$.

All electronic calculations were carried out using the MOLPRO suite of $a b$ initio programs. ${ }^{61}$ The potential energy surfaces, as well as the electric dipole and transition moment functions, have been computed using internally contracted multireference configuration interaction (MRCI) wave functions ${ }^{62,63}$ with single and double excitations. All configurations that contribute more than 5\% to the complete active space self-consistent field (CASSCF) wave function are used as reference configuration in the MRCI calculation. The Davidson size consistency correction ${ }^{64}(+Q)$ was applied.

State average multiconfiguration (SA-MC) orbitals were obtained with all the configurations that contribute more than $5 \%$ to the state average CASSCF (SA-CASSCF) and to preliminary $\mathrm{MRCI}+Q$ calculations. The lowest $1 a^{\prime}$ molecular orbital ( $\sim 1 s$ orbital on fluorine) has been kept frozen in all calculations (but optimized doubly occupied at the CASSCF). In the entrance channel, the four electronic states of interest are well separated from other electronic excited states. However, in the products region the ionic-covalent curve crossing (see Figs. 1 and 2) causes strong orbital changes and makes difficult the CASSCF optimization. This strong change may lead to discontinuities in the potential energy surfaces. To avoid this problem, in all the calculations, all the configurations contributing for the ground electronic state in any geometry have been included. In addition, all configurations contributing to the excited electronic states in the reactant channel have also been included. After the curve crossing all the excited electronic states correlate with the LiF excited states, which are very high in energy. Therefore, in the energy range of interest, it is not important to 

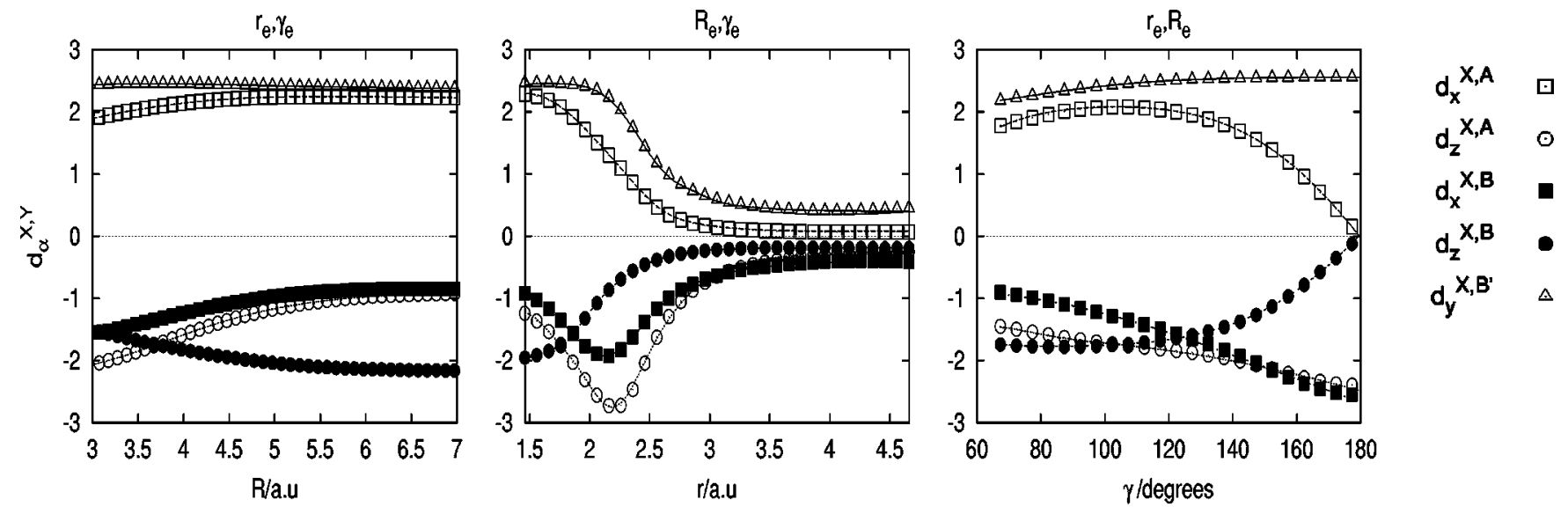

FIG. 3. Transition electric dipole moment components for the $A, B, B^{\prime} \leftarrow X$ transitions (in a.u.)

include new configurations to properly describe the high energy, which avoids the possible discontinuities of the PES.

All calculations were performed using the $C_{s}$ group, as usual. In order to consider the $\Pi$ degeneration (calculated in $C_{s}$ as $A^{\prime}, A^{\prime \prime}$ ), we have selected the active space to maintain the asymptotic degeneracy of the $2 p$ lithium orbitals, including 11 electrons in 10 active orbitals $\left(2 a^{\prime}-9 a^{\prime}, 1 a^{\prime \prime}\right.$ $-2 a^{\prime \prime}$ ), and optimizing the ${ }^{2} A^{\prime}$ and ${ }^{2} A^{\prime \prime}$ states together. This makes the states of $\Pi$ symmetry practically degenerate, with a negligible difference at the CASSCF level, and of the order of $20 \mathrm{~cm}^{-1}\left(0.1 \mathrm{~m} E_{h}\right)$ for MRCI $+Q$ energies. The main reference configurations in the SA-MC as well as in the MRCI calculation are

$$
\psi_{n}=\left|\left(1 a^{\prime}\right)^{2}\left(2 a^{\prime}\right)^{2}\left(3 a^{\prime}\right)^{2}\left(4 a^{\prime}\right)^{2}\left(1 a^{\prime \prime}\right)^{2}\left(5 a^{\prime}\right)^{2}\left(n a^{\prime}\right)^{1}\right|
$$

with $n=6,7,8$, and 9 , for the ${ }^{2} A^{\prime}$ states, and

$$
\psi_{m}=\left|\left(1 a^{\prime}\right)^{2}\left(2 a^{\prime}\right)^{2}\left(3 a^{\prime}\right)^{2}\left(4 a^{\prime}\right)^{2}\left(1 a^{\prime \prime}\right)^{2}\left(5 a^{\prime}\right)^{2}\left(m a^{\prime \prime}\right)^{1}\right|
$$

with $m=2$ and 3 , for the ${ }^{2} A^{\prime \prime}$ state. These reference configurations are the most important in the nuclear geometries of interest in this work. Nevertheless we have used 23 and 5 reference configurations for $A^{\prime}$ and $A^{\prime \prime}$ states, respectively. The reference configurations give a final contribution to the MRCI wave function of more than $97 \%$ for all the roots, with a maximum number of contracted (uncontracted) configurations of the order of 1400000 (5800 000) and 380000 (2000 000) for the ${ }^{2} A^{\prime}$ and ${ }^{2} A^{\prime \prime}$ symmetries, respectively.

The $a b$ initio calculations have been performed on an extended geometry grid, that covers the nuclear region of interest. For the grid we have used bond coordinates $\left(R_{\mathrm{HF}}, R_{\mathrm{LiF}}, \theta=\mathrm{Li} \hat{\mathrm{F}} \mathrm{H}\right)$, in the range:

$$
\begin{aligned}
& R_{\mathrm{HF}}=1.1-10 a_{0} \quad \text { (at } 35 \text { intervals), } \\
& R_{\mathrm{LiF}}=2.0-20 a_{0} \text { (at } 30 \text { intervals), } \\
& \theta=0-180^{\circ} \quad \text { (at } 30 \text { intervals). }
\end{aligned}
$$

Because the number of points of this grid is excessive (31 500), calculations were performed on a selected subset of about 6500 points. The selection of the points was based on previous calculations, ${ }^{45,44}$ always keeping the main goal of describing the van der Waals well and the saddle point regions.

\section{The LiHF transition dipole moment}

The transition electric dipole moments have been calculated using the MRCI wave functions, in the region of interest, being approximately expressed as a simple sum of monodimensional functions as

$$
d_{\alpha}^{X Y}(r, R, \gamma)=d_{\alpha, e}^{X Y}+d_{\alpha, r}^{X Y}(r)+d_{\alpha, R}^{X Y}(R)+d_{\alpha, \gamma}^{X Y}(\gamma),
$$

where $X$ refers to the ground electronic state $\left(X^{2} A^{\prime}\right)$, and $Y$ to the excited states- $A\left({ }^{2} A^{\prime}\right), B\left({ }^{2} A^{\prime}\right)$, or $B^{\prime}\left({ }^{2} A^{\prime \prime}\right)$. In this expression $\mathbf{r}$ and $\mathbf{R}$ reactant Jacobi vectors are used, where $\mathbf{r}$ is the HF internuclear vector and $\mathbf{R}$ joins the HF center-ofmass to the Li atom, with $\gamma$ being the angle between them. A body-fixed frame is used in which the $z$ axis lies along $\mathbf{R}$ and the three atoms are in the $x-z$ plane. Finally, $\alpha=x, y, z$ and $d_{\alpha, e}^{X Y}=d_{\alpha}^{X Y}\left(r_{e}, R_{e}, \gamma_{e}\right)$ corresponds to the minimum of the well of the $X\left({ }^{2} A^{\prime}\right)$ state. In Eq. (5), the monodimensional $d_{\alpha, r}^{X Y}(r)$ functions have been described by

$$
d_{\alpha, r}^{X Y}(r)=\sum_{i=1}^{3} a_{i} \exp \left[-b_{i}\left(r-r_{e}\right)^{2 c_{i}}\right],
$$

where the $a_{i}, b_{i}, c_{i}$ parameters have been fitted for each $X Y$ transition and each Cartesian component $\alpha$, with $c_{i}$ being normally integers. Similar expressions holds for the $R$ and $\gamma$ cases.

Note that the calculated transition dipole moments can change sign from one geometry to a different one because of a phase change of one of the wave functions involved. In order to avoid this sign change, we have calculated the overlap between the wave functions at the different geometries, and corrected the sign when necessary.

The transition dipole moments obtained, see Fig. 3, are important for the three final electronic states according to the usual selection rules imposed by their symmetry: $X-A$ and $X-B$ correspond to transitions in the $x-z$ plane, while the $X-B^{\prime}$ is out of the plane with $d_{y}$ being the only nonzero term. In order to compare with our previous results on the $X-A$ transition dipole moments (Fig. 5 of Ref. 44, where there is a mistake in the tics: $\gamma$ should vary from $60^{\circ}$ to $180^{\circ}$ instead of from $30^{\circ}$ to $100^{\circ}$ ), we have selected in Fig. 3 the same sign criteria. 
If the four states considered here would be approximately described by electronic configurations built as a simple product of a function for lithium atom in the ${ }^{2} S$ or ${ }^{2} P$ states and the $\operatorname{HF}\left({ }^{1} \Sigma^{+}\right)$functions, the transition selection rule will correspond to those of the isolated lithium atom. In such case, considering a monoelectronic atom, $B^{\prime} \leftarrow X$ will be the only allowed transition. However, clearly this is not the situation here. The reason is that the electronic structure is much more complicated because of the crossing with the ionic $\mathrm{Li}^{+}-\mathrm{HF}^{-}$configurations, which are the responsible for the $A, B \leftarrow X$ transitions in the region of the wells. In addition to the avoided curve crossing in Fig. 1 giving rise to the product channel, there are some other curve crossing and/or conical intersections among the excited electronic states.

The three excited electronic states, $A^{2} A^{\prime}, B^{2} A^{\prime}$, and $B^{\prime 2} A^{\prime \prime}$ in the $C_{s}$ point group, correlating with the excited ${ }^{2} P$ state of lithium become the ${ }^{2} \Sigma^{+}$and ${ }^{2} \Pi$ states in the $C_{\infty v}$ point group. This split of the ${ }^{2} P$ state is responsible of a conical intersection, as shown in Fig. 4(a) for collinear configurations corresponding to the equilibrium $\mathrm{HF}$ distance. At short $\mathrm{LiF}$ distances $\left(R_{\mathrm{LiF}}<4\right.$ a.u. $)$ the $B^{2} A^{\prime}$ and $B^{\prime 2} A^{\prime \prime}$ states are degenerate, and at longer $\mathrm{LiF}$ distances $\left(R_{\mathrm{LiF}}\right.$ $>4$ a.u.) the $A^{2} A^{\prime}$ and $B^{\prime 2} A^{\prime \prime}$ are the degenerate ones. On the other hand, we show, too, in Fig. 4(b), the potential energy cut along the Jacobi $\gamma$ angle for a configuration near the minimum of the $A^{2} A^{\prime}$ electronic state. This cut shows that there is a change in the degenerate states going from one collinear geometry corresponding to $\mathrm{Li}-\mathrm{F}-\mathrm{H}\left(\gamma=180^{\circ}\right)$ to the other one $\mathrm{F}-\mathrm{H}-\mathrm{Li}\left(\gamma=0^{\circ}\right)$. The treatment of all these crossings and the possible diabatization deserves a very detailed study, which will be done in a forthcoming work.

\section{GLOBAL POTENTIAL ENERGY SURFACES}

Here each adiabatic PES is fitted independently. Each global PES (GPES) is obtained using the fitting procedure developed by two of the authors (A.A. and M.P.) ${ }^{65}$ briefly described here. A modified many-body expansion is used:

$$
\begin{aligned}
V_{\mathrm{LiHF}}= & \sum_{A}^{3} V_{A}+\sum_{A B}^{3} V_{A B}^{(2)}\left(R_{A B}\right) \\
& +\sum_{L}^{L_{\max }} V_{A B C}^{(3) L}\left(R_{A B}, R_{A C}, R_{B C}\right),
\end{aligned}
$$

where several three-body terms $\left(L_{\max }>1\right)$ are considered to obtain a better fit. ${ }^{66}$ These three-body terms are expanded as

$$
V_{A B C}^{(3) L}\left(R_{A B}, R_{A C}, R_{B C}\right)=\sum_{i j k}^{K} d_{i j k}^{L} \rho_{A B}^{i} \rho_{A C}^{j} \rho_{B C}^{k},
$$

where the variables $\rho_{A B}$ are modified forms of the Rydberg functions,

$$
\rho_{A B}\left(R_{A B}\right)=R_{A B} e^{-\beta_{A B}^{(N)} R_{A B}} \quad\left(\beta_{A B}^{(N)}>0\right)
$$

and similarly for $\rho_{A C}$ and $\rho_{B C}$. These functions depend on the internuclear distances $R_{A B}, R_{A C}$, or $R_{B C}$, and tend to zero when the corresponding distance goes to zero or to infinity. Such behavior avoids the appearance of artificial
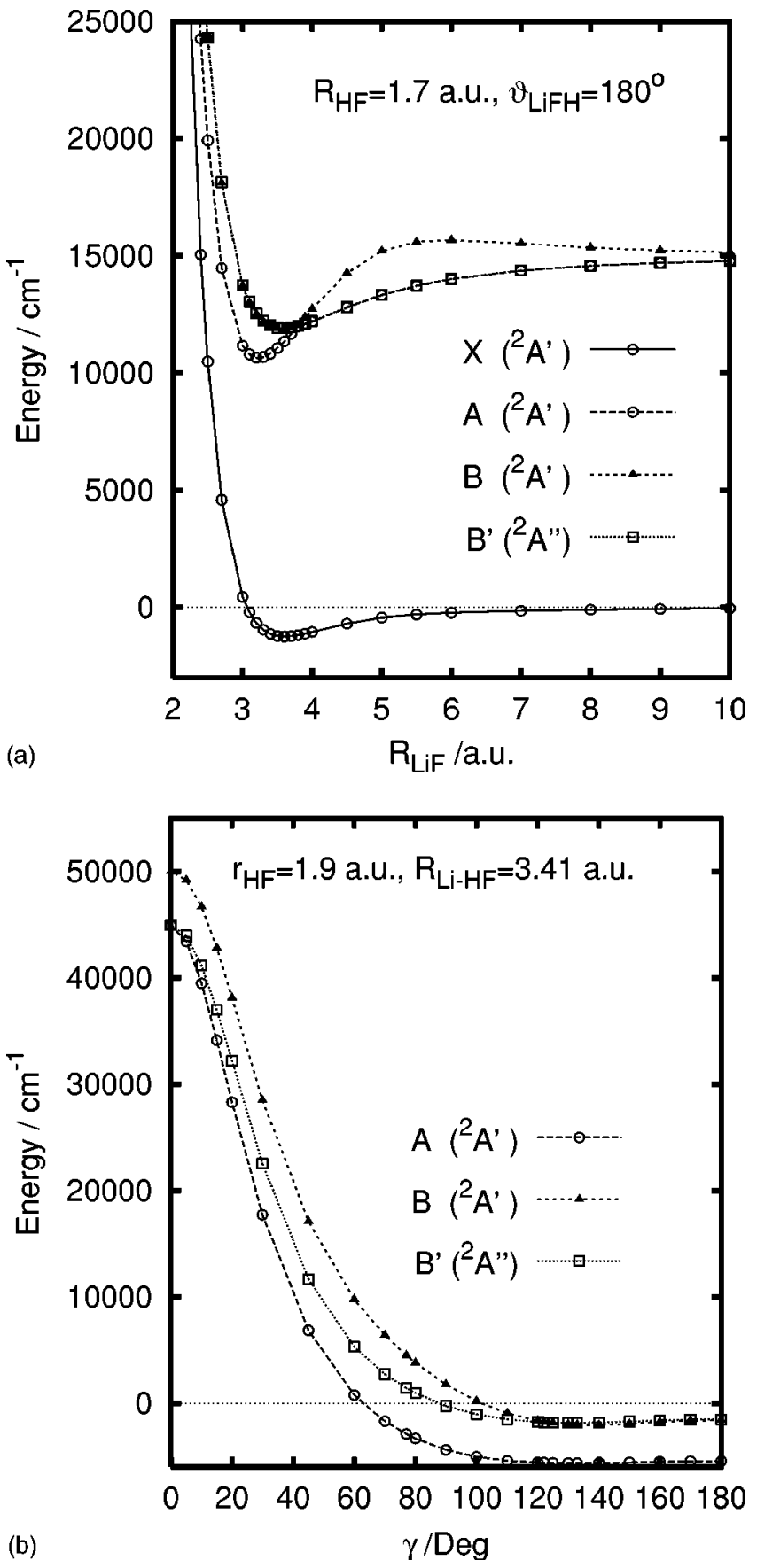

FIG. 4. One-dimensional cut that shows several conical intersections. (a) Collinear cut along $R_{\mathrm{LiF}}$ with $r_{\mathrm{HF}}$ fixed at $1.7 a_{0}$. (b) Cut along the Jacoby $\gamma$ angle.

minima at short distances and makes that the total PES is described, at long distances, by the sum of two-body terms, $V_{A B}^{(2)}$, and atomic energies, $V_{A}$.

The description of the two-body terms is done by decomposing as $V_{A B}=V_{\text {short }}+V_{\text {long }}$, with

$$
\begin{aligned}
& V_{\text {short }}=c_{\mathrm{O}} \frac{e^{-\alpha R_{A B}}}{R_{A B}}, \\
& V_{\text {long }}=\sum_{i}^{I} c_{i} \rho_{A B}^{i},
\end{aligned}
$$

where the $\rho_{A B}$ functions are given in Eq. (8). 
TABLE II. Errors in $\mathrm{cm}^{-1}$ of the GPES fits for the $X, A, B$, and $B^{\prime}$ states of LiHF with respect to the calculated ab initio points. The energy ranges are referred relative to the minimum of $\mathrm{HF}$ of their corresponding $\mathrm{Li}+\mathrm{HF}$ asymptote.

\begin{tabular}{ccrr}
\hline \hline & Energy range & $\mathrm{N}_{\text {geom }}$ & $\mathrm{rms}$ \\
\hline$X$ state & & & \\
& $E<60000$ & 6104 & 399 \\
& $16000<E<20000$ & 272 & 352 \\
$8000<E<16000$ & 954 & 213 \\
& $0<E<8000$ & 2301 & 155 \\
& $-2000<E<0$ & 1190 & 89 \\
A state & & & \\
& $E<30000$ & 4703 & 415 \\
& $0.0<E<8000$ & 1110 & 341 \\
& $-3000<E<0$ & 930 & 251 \\
$B$ state & $-6000<E<-3000$ & 976 & 180 \\
& & & \\
& $E<30000$ & 4812 & 810 \\
& $0.0<E<8000$ & 1690 & 317 \\
$B^{\prime}$ state & $-3500<E<0$ & 792 & 56 \\
& & & \\
& $E<30000$ & 5083 & 914 \\
& $0.0<E<8000$ & 1318 & 892 \\
& $-3500<E<0$ & 1259 & 21 \\
\hline \hline
\end{tabular}

The linear parameters $d_{i j k}^{L}$ and the nonlinear parameters $\beta_{A B}^{(3) L}$ are determined by fitting the calculated $a b$ initio energies of the above-described triatomic. The diatomic equilibrium distance, dissociation energy, frequency, and anharmonicity constant are compared with those obtained in previous fits of the ground state of the LiHF system and vibrational energies for the ground electronic state of the three diatomic fragments are compared with the "experimental" ones, all showing a rather good agreement. The rms error of the four different fits and in different energy ranges is shown in Table II, being lower than $180 \mathrm{~cm}^{-1}$ in the energy range of interest in this work.

The fit for each electronic state is done relative to its corresponding $\mathrm{Li}+\mathrm{HF}$ asymptote at the minimum of the $\mathrm{HF}$ well. The $a b$ initio $\operatorname{Li}\left({ }^{2} P \leftarrow{ }^{2} S\right)$ excitation energy is larger than the experimental value by $\approx 50 \mathrm{~cm}^{-1}$. In order to compare with the experimental spectrum, ${ }^{26}$ here we use the experimental value of $14904 \mathrm{~cm}^{-1}$. In addition, several calculations allowed us to estimate the basis set superposition error to be of the order of $100 \mathrm{~cm}^{-1}$, for the $A$ state which presents the deepest well. In order to get a better agreement with the experimental spectrum, the GPES for the $A$ state is corrected as follows. Equation (6) is rewritten as,

$$
V_{\mathrm{LiHF}}^{A}=V_{\mathrm{Li} *}+V_{\mathrm{HF}}\left(R_{\mathrm{HF}}\right)+W_{\mathrm{LiHF}}^{A},
$$

where

$$
W_{\mathrm{LiHF}}^{A}=V_{\mathrm{LiF}}+V_{\mathrm{HLi}}+\sum_{L}^{L_{\max }} V_{A B C}^{(3) L}\left(R_{A B}, R_{A C}, R_{B C}\right),
$$

and this $W_{\mathrm{LiHF}}^{A}$ is multiplied by a factor of $\approx 0.94$ in order to reduce the overestimated dissociation energy of the $A$ state well. This factor, together with the use of the experimental $\mathrm{Li}\left({ }^{2} P \leftarrow{ }^{2} S\right)$ excitation energy are the only two no ab initio
$\mathrm{R}_{\mathrm{HF}}=2.41$ a.u.
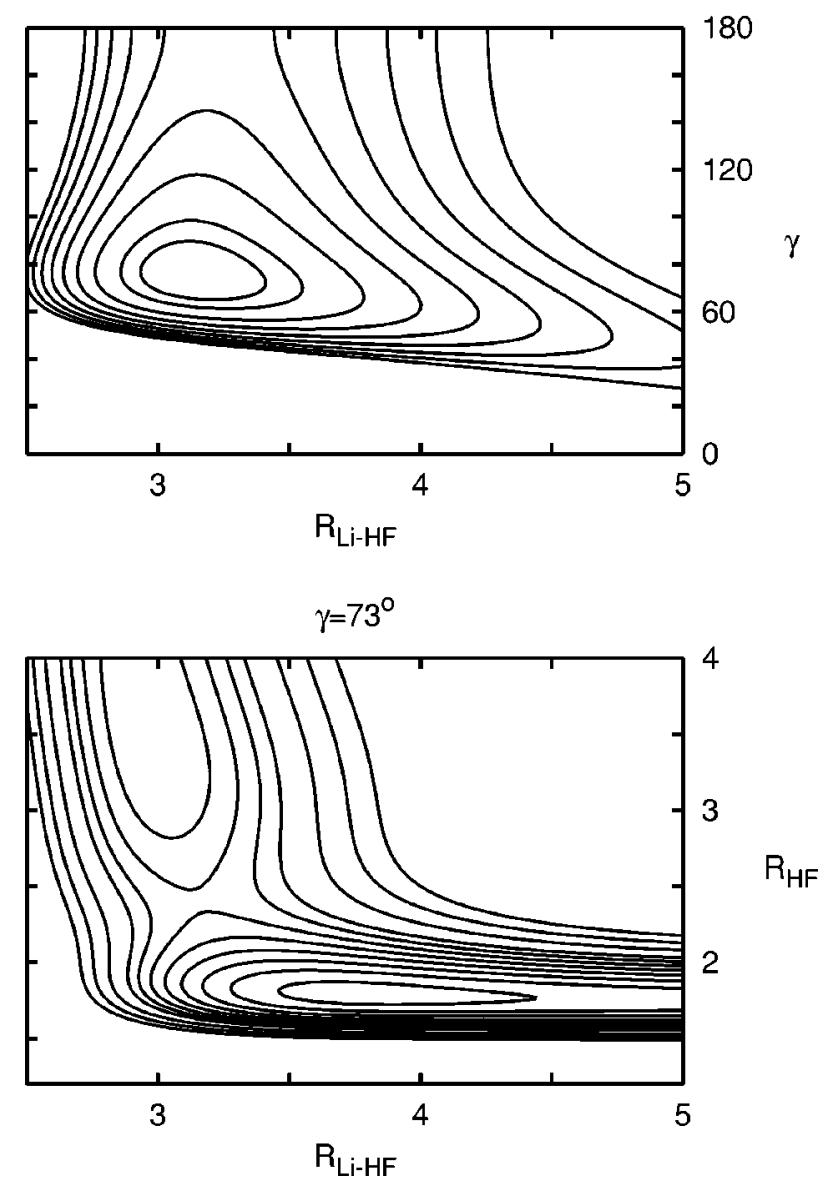

FIG. 5. Contour plots of the $\operatorname{LiHF}(X)$ PES as function of Jacobi coordinates. The energy contours start in 2000 and -500 with steps of 1000 and 500 for the top and bottom panels, respectively. Energy is in $\mathrm{cm}^{-1}$ referred to the minimum of $\mathrm{HF}$ well in the $\mathrm{Li}+\mathrm{HF}$ asymptote.

parameters of the GPESs used in this work. In what follows we will refer to this corrected version of the $A$ state.

The GPES for the $\operatorname{LiHF}(X)$ presents a late barrier connecting $\operatorname{Li}\left({ }^{2} S\right)+\mathrm{HF}\left({ }^{1} \Sigma^{+}\right)$reactants with $\mathrm{H}\left({ }^{2} S\right)$ $+\operatorname{LiF}\left(X^{1} \Sigma^{+}\right)$products, while the $\operatorname{HLi}\left(X^{1} \Sigma^{+}\right)$products appear at much higher energies of $\approx 28000 \mathrm{~cm}^{-1}$. Also the first electronic excitation of the $\mathrm{H}$ or LiF products is so high in energy that the GPESs for the $A, B$, and $B^{\prime}$ states can be considered to be nonreactive and their main feature is a well in the reactant channel. We shall then describe them separately comparing with the previous available surfaces in the following.

In Fig. 5 several contour plots of the GPES for the $\operatorname{LiHF}\left(X^{2} A^{\prime}\right)$ are shown as a function of the $r, R$, and $\gamma$ Jacobi coordinates, focusing on the transition state region. The stationary points are shown in Table III and compared with some previous GPES for this state. They all show a well in the entrance channel, $\approx 2000 \mathrm{~cm}^{-1}$ deep at $\approx 110^{\circ}$, a late barrier, with the HF distance rather elongated with respect to the free HF equilibrium distance, and a rather shallow well in the $\mathrm{H}+\mathrm{LiF}$ products channel. All the surfaces used in Table III show a very good qualitative agreement, but it is notorious the excellent agreement with the nowadays more accurate APW-GPES produced for $\operatorname{LiHF}(X),{ }^{43}$ and with the PESs 
TABLE III. Comparison of geometries and energies of the stationary points for several ab initio fitted LiHF global PESs. Energies, in $\mathrm{cm}^{-1}$, are relative to the $\mathrm{Li}\left({ }^{2} S\right)+\mathrm{HF}$ asymptote.

\begin{tabular}{|c|c|c|c|c|c|c|}
\hline & $\mathrm{CS}^{\mathrm{b}}$ & $\mathrm{CM}^{\mathrm{c}}$ & $\mathrm{SRPA}^{\mathrm{d}}$ & $\mathrm{JHTP}^{\mathrm{e}}$ & $\mathrm{APW}^{\mathrm{f}}$ & Present \\
\hline & \multicolumn{6}{|c|}{$\operatorname{LiHF} X^{2} A^{\prime}$} \\
\hline Reactants & & & & & & \\
\hline$r_{\mathrm{HF}}^{\mathrm{a}}$ & 1.76 & 1.73 & 1.74 & 1.733 & 1.736 & 1.741 \\
\hline Energy & 0.0 & 0.0 & 0.0 & 0.0 & 0.0 & 0.0 \\
\hline \multicolumn{7}{|c|}{ Reactant van der Waals well } \\
\hline$r_{\mathrm{LiF}}^{\mathrm{a}}$ & 3.68 & 3.59 & 3.552 & 3.56 & 3.555 & 3.558 \\
\hline$r_{\mathrm{HF}}{ }^{\mathrm{a}}$ & 1.78 & 1.76 & 1.775 & 1.76 & 1.758 & 1.756 \\
\hline$\theta$ & $109^{\circ}$ & $114^{\circ}$ & $107.4^{\circ}$ & $107^{\circ}$ & $104.8^{\circ}$ & $110.0^{\circ}$ \\
\hline Energy & -1613 & -2100 & -2251 & -1940 & -1959 & -1942 \\
\hline \multicolumn{7}{|c|}{ Saddle point } \\
\hline$r_{\mathrm{LiF}}^{\mathrm{a}}$ & 3.21 & 3.12 & 3.197 & 3.10 & 3.161 & 3.174 \\
\hline$r_{\mathrm{HF}}^{\mathrm{a}}$ & 2.44 & 2.48 & 2.457 & 2.62 & 2.397 & 2.413 \\
\hline$\theta$ & $74^{\circ}$ & $74^{\circ}$ & $71.4^{\circ}$ & $72.8^{\circ}$ & $72.9^{\circ}$ & $73.4^{\circ}$ \\
\hline Energy & 3469 & 2500 & 1880 & 1992 & 1783 & 2019 \\
\hline \multicolumn{7}{|c|}{ Product van der Waals well } \\
\hline$r_{\mathrm{LiF}}^{\mathrm{a}}$ & $\ldots$ & $\cdots$ & 2.997 & 2.98 & 2.979 & 3.029 \\
\hline$r_{\mathrm{HF}}^{\mathrm{Lu}}{ }^{\mathrm{a}}$ & $\cdots$ & $\cdots$ & 5.139 & 3.90 & 4.661 & 3.704 \\
\hline$\theta$ & $\cdots$ & $\cdots$ & $90.9^{\circ}$ & $68.4^{\circ}$ & $69.7^{\circ}$ & $63.7^{\circ}$ \\
\hline Energy & $\cdots$ & $\cdots$ & 68 & 1210 & 540 & 948 \\
\hline \multicolumn{7}{|l|}{ Products } \\
\hline$r_{\mathrm{LiF}}^{\mathrm{a}}$ & 3.03 & 2.95 & 3.00 & 2.955 & 2.959 & 2.970 \\
\hline Energy & 0.13 & 0.17 & 661 & 1412 & 944 & 1501 \\
\hline \multirow{2}{*}{\multicolumn{7}{|c|}{$\operatorname{LiHF} A^{2} A^{\prime}$}} \\
\hline & & & & & & \\
\hline$r_{\mathrm{HF}}^{\mathrm{a}}$ & $\cdots$ & $\cdots$ & 1.74 & 1.733 & $\cdots$ & 1.741 \\
\hline Energy $^{a}$ & $\cdots$ & $\cdots$ & 16565 & 14905 & $\cdots$ & 14950 \\
\hline \multicolumn{7}{|c|}{ Reactant van der Waals well } \\
\hline$r_{\mathrm{LiF}}^{\mathrm{a}}$ & $\cdots$ & $\cdots$ & 3.160 & 3.34 & $\cdots$ & 3.396 \\
\hline$r_{\mathrm{HF}}{ }^{\mathrm{a}}$ & $\cdots$ & $\cdots$ & 1.984 & 1.82 & $\cdots$ & 1.868 \\
\hline$\theta$ & $\cdots$ & $\cdots$ & $180^{\circ}$ & $122^{\circ}$ & $\cdots$ & $125^{\circ}$ \\
\hline \multirow[t]{2}{*}{ Energy } & $\cdots$ & $\cdots$ & 9143 & 9792 & $\cdots$ & 9622 \\
\hline & \multicolumn{6}{|c|}{$\operatorname{LiHF} B^{2} A^{\prime}$} \\
\hline \multicolumn{7}{|c|}{ Reactant van der Waals well } \\
\hline$r_{\mathrm{LiF}}^{\mathrm{a}}$ & $\cdots$ & $\cdots$ & 3.575 & $\cdots$ & $\cdots$ & 3.535 \\
\hline$r_{\mathrm{HF}}^{\mathrm{Lu}}{ }^{\mathrm{a}}$ & $\cdots$ & $\cdots$ & 1.750 & $\cdots$ & $\cdots$ & 1.761 \\
\hline$\theta$ & $\cdots$ & $\cdots$ & $140^{\circ}$ & $\cdots$ & $\cdots$ & $149^{\circ}$ \\
\hline \multirow[t]{2}{*}{ Energy } & $\cdots$ & $\cdots$ & 14400 & $\cdots$ & $\cdots$ & 11764 \\
\hline & \multicolumn{6}{|c|}{ LiHF $B^{\prime 2} A^{\prime \prime}$} \\
\hline \multicolumn{7}{|c|}{ Reactant van der Waals well } \\
\hline$r_{\mathrm{LiF}}^{\mathrm{a}}$ & $\cdots$ & $\cdots$ & 3.991 & $\cdots$ & $\cdots$ & 3.642 \\
\hline$r_{\mathrm{HF}}{ }^{\mathrm{a}}$ & $\cdots$ & $\cdots$ & 1.719 & $\cdots$ & $\cdots$ & 1.747 \\
\hline$\theta$ & $\cdots$ & $\cdots$ & $149^{\circ}$ & $\cdots$ & $\cdots$ & $131^{\circ}$ \\
\hline Energy & $\cdots$ & $\ldots$ & 14010 & $\ldots$ & $\cdots$ & 11638 \\
\hline
\end{tabular}

${ }^{a}$ All distances in bohr.

${ }^{\mathrm{b}}$ Reference 83.

${ }^{c}$ Reference 84 .

${ }^{\mathrm{d}}$ Reference 45

${ }^{\mathrm{e}}$ Reference 42.

${ }^{\mathrm{f}}$ Reference 43.

obtained by Burcl et al. ${ }^{53}$ To check the accuracy of the $X$-GPES for reactive collisions, which are very sensitive to the details of the potential, preliminary wave packet calculations have been made for $\operatorname{Li}+\operatorname{HF}(v=0,1)$ reactive cross sections, obtaining results in rather good agreement with those performed using the APW-GPES. ${ }^{43}$

Figure 6 shows contour plots of the $X, A, B$, and $B^{\prime}$ electronic states of LiHF with $r$ fixed at the corresponding equilibrium distances (see Table III), being $x=R \cos \gamma$ and $y=R \sin \gamma$. All the states present a rather similar $r_{e}$, being slightly larger than that of the free HF. The well of the $A$ state being the deepest one also presents a longer $r_{e}$. The equilibrium $R_{e}$ values, however, are shorter for the $A$ state, being rather similar among the rest of the electronic states. Nevertheless, all the radial equilibrium distances are rather similar among the different electronic states. The larger difference arises among the angular dependence of each PES. The equilibrium angles are $110^{\circ}, 125^{\circ}, 150^{\circ}$, and $130^{\circ}$ for the $X, A, B$, and $B^{\prime}$ electronic states, respectively. Such differences have important implications in the spectrum, as 


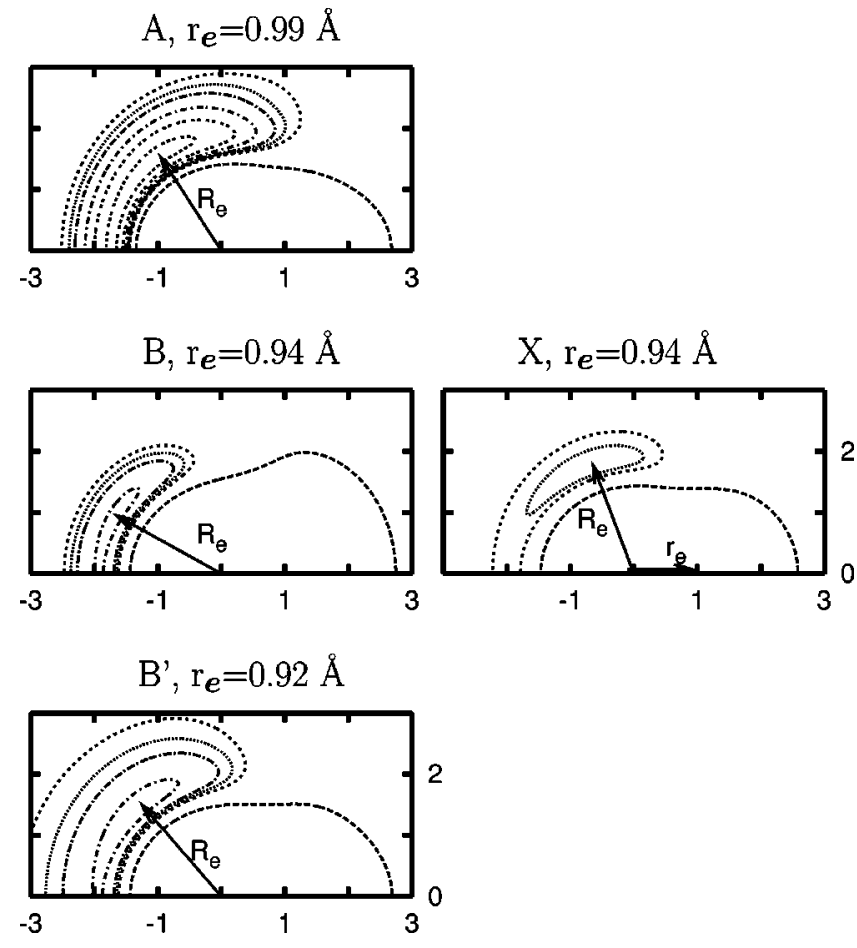

FIG. 6. Contour plots of the LiHF PESs for the $X, A, B$, and $B^{\prime}$ states in the vicinity of the reactant channel well with the HF distance $r$ frozen to its equilibrium distance. $x=R \cos \gamma$ and $y=R \sin \gamma$. The energy contours are $-5000,-4000,-3000,-1500,-1000$, and $5000 \mathrm{~cm}^{-1}$, referred to the minimum of $\mathrm{HF}$ well in the corresponding $\mathrm{Li}+\mathrm{HF}$ asymptote, and distances in $\AA$.

will be discussed in the following. Another striking feature is the small dependence of the PES as a function of the angle between the equilibrium angle and $180^{\circ}$. Such situation is especially apparent for the first excited state $\left(A^{2} A^{\prime}\right)$, as shown in Fig. 4(b): the difference between the minimum at $130^{\circ}$ and at $120^{\circ}$ is only of $0.5 \mathrm{~cm}^{-1}$, increasing up to about $100 \mathrm{~cm}^{-1}$ for $180^{\circ}$.

The stationary points of the $A$ state are in rather good agreement with those of Jasper et al., ${ }^{42}$ presenting a less deep well than the present one. These authors do not report analytical fits of the $B$ and $B^{\prime}$ states, and there is no detailed description of these surfaces. Some semiquantitative idea of those two PESs can be extracted from Figs. 8 to 11 of Ref. 42. A cut for $\mathrm{Li}-\mathrm{F}-\mathrm{H}$ bond angle of $110^{\circ}$ and $r_{\mathrm{HF}}$ $=1.73$ a.u., in Fig. 10 of Ref. 42, the $B^{\prime}$ well seems to be nearly as deep as that of the $A$ state. By contrast, in this work the $B^{\prime}$ curve is in between the $A$ and $B$ potential curves, at that configuration. We have performed several calculations changing the atomic basis set, the chosen configurations, etc., and the relative position of the PESs remained nearly constant with the results described earlier in this work. In addition, the $B$ and $B^{\prime}$ PESs of Ref. 42 were obtained with a reduced number of configurations, as compared with the number used for the $X$ and $A$ states. Without more knowledge about the calculations of Ref. 42 and the extensive analysis performed in this work, we have to conclude that our results are essentially correct.

The GPESs obtained in this work are considerably improved with respect to the previous work for the $A^{44}$ and $B$ and $B^{\prime}$ states: $^{45}$ the $a b$ initio calculations are more accurate (because the improvement in the atomic basis set and the electronic configuration selected), the number of calculated points is larger, and the quality of the fit is also better. Four different files containing the fits, as FORTRAN subroutines, for the electronic states here considered are placed in the electronic depository EPAPS. ${ }^{67}$

\section{SPECTRA CALCULATIONS}

\section{A. Bound state calculations}

Using reactant Jacobi coordinates in a body-fixed frame, as described earlier, the bound eigenstates for each of these four electronic states, $\chi_{\alpha, \sigma}$ with $\alpha=X, A, B$, and $B^{\prime}$, are expanded as

$\Psi_{k}^{J M \epsilon \alpha}(\mathbf{r}, \mathbf{R})=\sum_{v s j \Omega} A_{v s j \Omega}^{J \epsilon \alpha k} \frac{\varphi_{v}(r)}{r} \frac{\phi_{s}(R)}{R} \mathcal{W}_{M \Omega}^{J \epsilon \epsilon} Y_{j \Omega}(\gamma, 0)$,

where the angular functions, defined as

$$
\begin{aligned}
\mathcal{W}_{M \Omega}^{J \epsilon \alpha}= & \sqrt{\frac{2 J+1}{16 \pi^{2}\left(1+\delta_{\Omega 0}\right)}}\left[D_{M, \Omega}^{J *}(\phi, \theta, \chi) \chi_{\alpha, \sigma}\right. \\
& \left.+\epsilon \sigma(-1)^{J+\Omega} D_{M,-\Omega}^{J *}(\phi, \theta, \chi) \chi_{\alpha, \sigma}\right]
\end{aligned}
$$

correspond to a well-defined total angular momentum, $J$, and parity under inversion of spatial coordinates, $\epsilon . M$ and $\Omega$ are the projections of the total angular momentum in the spacefixed and body-fixed frames, respectively. $D_{M, \Omega}^{J *}$ are Wigner rotation matrices, $Y_{j, \Omega}$ are normalized associated Legendre functions, ${ }^{68}$ and $\chi_{\alpha, \sigma}$ electronic wave functions (with eigenvalue $\sigma$ of the $\sigma_{x z}^{\text {bf }}$ operator, the reflection through the bodyfixed $x-z$ plane).

The potential-optimized $\varphi_{v}(r)$ and $\phi_{s}(R)$ radial basis set functions in Eq. (11) are solutions of a one-dimensional Hamiltonian obtained numerically using some reference potential. The reference potential corresponds to the equilibrium configuration of all coordinates except that of interest. The HF stretch is by far the largest one for the two states (being $\approx 3500$ and $2650 \mathrm{~cm}^{-1}$ for the $X, B, B^{\prime}$, and $A$ states, respectively, while it is 4000 for free HF), and only a few functions are required to get a good convergence (here $v$ $=0, \ldots, 6$ were used). The frequency of the $R$ mode is much lower and many more functions are needed. The number of $\phi_{s}(R)$ radial functions obtained with a single reference potential is insufficient to converge the calculation of very excited states, close to the dissociation limit. To avoid this problem, several reference potentials are used, and the corresponding functions are orthonormalized. Basis set functions are added to converge eigenvalues up to $-1000 \mathrm{~cm}^{-1}$ in the $A$ state and up to $2048 \mathrm{~cm}^{-1}$ for the $B$ and $B^{\prime}$ states [being $2048 \mathrm{~cm}^{-1}$ the first $\operatorname{Li}\left({ }^{2} P\right)+\mathrm{HF}(v=0)$ dissociation energy] so that the error is better than $\left.1-2 \mathrm{~cm}^{-1}\right)$. In the 
TABLE IV. Vibrational levels of LiHF obtained for $J=0$, and $X, A, B$ and $B^{\prime}$ electronic states. Energies are referred to the minimum of HF in their corresponding $\mathrm{Li}+\mathrm{HF}$ asymptotes, in $\mathrm{cm}^{-1}$. The $(v, n, b)$ approximated quantum numbers correspond to $\mathrm{HF}, \mathrm{Li}-\mathrm{HF}$ stretching, and $\mathrm{L}-\mathrm{HF}$ bending, respectively.

\begin{tabular}{|c|c|c|c|c|c|c|c|c|c|c|c|c|c|c|c|c|}
\hline $\begin{array}{c}\text { State, } \\
n_{\text {vib }}\end{array}$ & $v$ & $n$ & $b$ & $\mathbf{X}$ & $v$ & $n$ & $b$ & $\mathbf{A}$ & $v$ & $n$ & $b$ & B & $v$ & $n$ & $b$ & $\mathbf{B}^{\prime}$ \\
\hline 1 & 0 & 0 & 0 & 319.51 & 0 & 0 & 0 & -3575.55 & 0 & 0 & 0 & -784.74 & 0 & 0 & 0 & -967.78 \\
\hline 2 & 0 & 0 & 1 & 615.10 & 0 & 0 & 1 & -3373.59 & 0 & 1 & 0 & -373.13 & 0 & 0 & 1 & -672.67 \\
\hline 3 & 0 & 1 & 0 & 674.50 & 0 & 0 & 2 & -3095.13 & 0 & 0 & 1 & -354.55 & 0 & 1 & 0 & -633.29 \\
\hline 4 & 0 & 0 & 2 & 871.72 & 0 & 1 & 0 & -3042.93 & 0 & 2 & 0 & 32.65 & 0 & 1 & 1 & -351.67 \\
\hline 5 & 0 & 1 & 1 & 943.49 & 0 & 1 & 1 & -2847.26 & 0 & 1 & 1 & 59.18 & 0 & 2 & 0 & -322.75 \\
\hline 6 & 0 & 2 & 0 & 992.98 & 0 & 0 & 3 & -2720.24 & 0 & 0 & 2 & 221.93 & 0 & 0 & 2 & -267.38 \\
\hline 7 & 0 & 0 & 3 & 1158.42 & 0 & 2 & 1 & -2592.43 & 0 & 3 & 0 & 424.93 & 0 & 3 & 0 & -53.96 \\
\hline 8 & 0 & 1 & 2 & 1203.21 & 0 & 1 & 2 & -2506.96 & 0 & 2 & 1 & 458.74 & 0 & 2 & 1 & -33.29 \\
\hline 9 & 0 & 2 & 2 & 1234.79 & 0 & 1 & 3 & -2358.22 & 0 & 1 & 2 & 631.10 & 0 & 1 & 2 & 52.94 \\
\hline 10 & 0 & 3 & 0 & 1276.07 & & & & -2316.32 & 0 & 4 & 0 & 801.30 & 0 & 4 & 0 & 213.70 \\
\hline 11 & 0 & 4 & 0 & 1418.82 & & & & -2171.75 & 0 & 3 & 1 & 842.79 & 0 & 0 & 3 & 227.12 \\
\hline 12 & & & & 1465.30 & & & & -2097.10 & 0 & 0 & 3 & 902.09 & 0 & 3 & 1 & 238.58 \\
\hline
\end{tabular}

following, a comparison with wave packet calculations will also serve as a test of the convergence of these calculations.

In Table IV the first energy levels for the four electronic states and $J=0$ are shown together with their assignment, and the contour plots of the wave functions for some of them are shown in Fig. 7. Approximated quantum numbers, $(v, n, b)$ are given which correspond to the HF vibration,
$\mathrm{Li}-\mathrm{HF}$ stretching, and $\mathrm{Li}-\mathrm{HF}$ bending, respectively, and are extracted from the analysis of the wave functions (see Fig. 7).

Concerning the ground electronic state, the first experimental evidence of the relatively deep well, of $\approx 2400 \mathrm{~cm}^{-1}$, in the entrance channel was provided by Loesch and Stienkemeier ${ }^{36}$ from backward glory scattering. The spec-


(b)

FIG. 7. Contour plots of the bound state amplitudes for the (a) $X$, (b) $A$, (c) $B$, and (d) $B^{\prime}$ states in the reactant channel well with the HF distance $r$ frozen to its equilibrium distance. $x=R \cos \gamma$ and $y=R \sin \gamma$, in $\AA$. 
troscopy experiments from this well, performed by Polanyi and co-workers, ${ }^{26}$ confirm its existence and its simulation is the subject of this work. This well appeared in nearly all $a b$ initio calculations and nearly at the same position, with slightly different dissociation energies. ${ }^{39,41,42,40,69,53,43}$ Bound state calculations ${ }^{52}$ performed on the APRL-2 PES ${ }^{41}$ yielded a $D_{0} \approx 2100, \omega_{r} \approx 3500$ (estimated by infrared excitation to the first HF overtone), $\omega_{R} \approx 353$, and $\omega_{\gamma} \approx 396\left(\right.$ all in $\mathrm{cm}^{-1}$ ), while using other surfaces Burcl et al. ${ }^{53}$ obtained values of $D_{0} \approx 1855-2033, \omega_{R} \approx 315-363$, and $\omega_{\gamma} \approx 362-395$ using different PESs. In this work, using the new PES, we obtain $D_{0} \approx 1729, \omega_{R} \approx 365$, and $\omega_{\gamma} \approx 296$, which are in rather good agreement with the previous values but also with the most accurate PES on the $\operatorname{LiHF}(X){ }^{43}$

There are few number of works on the $A$ electronic bound states. To our knowledge, there are only the adiabatic PES of Aguado et al. ${ }^{44}$ and coupled quasidiabatic PESs by Jasper et al. ${ }^{42}$ In this last surface there is no calculated bound state and, as discussed earlier, the $\operatorname{LiHF}(A)$ PES of Ref. 44 has an equilibrium angle of $180^{\circ}$ while the present one is of $\approx 125^{\circ}$. However, and because the new and old $\operatorname{LiHF}(A)$ PESs present a very weak dependence with the angle, the states are very similar and present a very similar structure when we compare the corresponding panels of Fig. 7 with those of Fig. 4 of Ref. 44. In the two cases the $\omega_{\gamma}$ is nearly half that of $\omega_{R}$ so that the first excitation in $R,(0,1,0)$, interacts strongly with the second excitation in $\gamma,(0,0,2)$ : odd bending excitations are well separated and can be clearly distinguished, while even bending excitations always interact with a stretching level, giving rise to a couple of mixed bending/stretching states. The frequencies associated with each vibrational mode are, approximately, $\omega_{\gamma} \approx 202 \mathrm{~cm}^{-1}$, $\omega_{R} \approx 526 \mathrm{~cm}^{-1}$, and $\omega_{r} \approx 3000 \mathrm{~cm}^{-1}$, reduced from that of free $\mathrm{HF}\left(\approx 4000 \mathrm{~cm}^{-1}\right)$ and that estimated for the $\operatorname{LiHF}(X)$ $\left(\approx 3500 \mathrm{~cm}^{-1}\right)$.

The only previous PESs for the $B$ and $B^{\prime}$ states are those of Ref. 45 and they are improved substantially here. These two surfaces must be degenerate at collinear configurations, but at bent geometries they differ substantially. Also these surfaces are quite anharmonic. The most striking thing is that there are states very excited in bending and not in stretching, indicating that this motion is pretty well separated from the others not only up to the energies shown but nearly up to dissociation, which is clearly not the case in the $X$ and $A$ states. Not only, while the $\omega_{R}$ decreases slightly with increasing energy, the energy separation among those states with pure bending excitation increases, see Table V. Such states will have important implications in the spectrum, as we will see in the following and deserve some special attention.

If the potential shows a well, the bending eigenvectors usually show the typical vibrational energy spacing decrease with increasing energy. In contrast, in a free rotation, the rotational energy is $B j(j+1)$, so that the energy spacing increases with energy. The real situation is somehow intermediate, as can be seen in Fig. 8, where the potential is shown for $r$ and $R$ frozen to their equilibrium values, which is a rather good approximation because of the net separation among the different modes. As a result, the energy difference
TABLE V. Energy differences between adjacent bending levels, $E_{(0,0, b)}$ $-E_{(0,0, b-1)}$, in $\mathrm{cm}^{-1}$, for $J=0$ and $B$ and $B^{\prime}$ electronic states compared with those corresponding to a monodimensional bending model with the distances frozen to their equilibrium values.

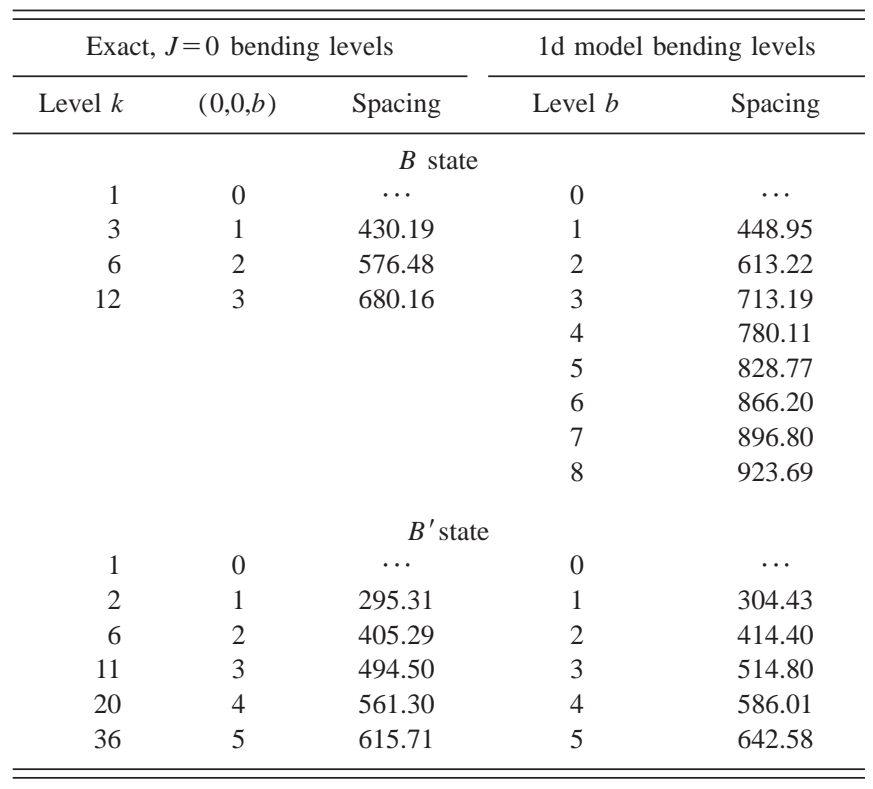

between these pure bending eigenvectors, also shown in Fig. 8 , increases. In fact, the energy spacings between different $(0,0, b)$ bending excitations of the $B$ and $B^{\prime}$ states compared with those quantities obtained using this simplified model, in Table V, are very good, up to $b=5$. From this level, the first dissociation energy, $\operatorname{Li}\left({ }^{2} P\right)+\operatorname{HF}(v=0)$, opens and the
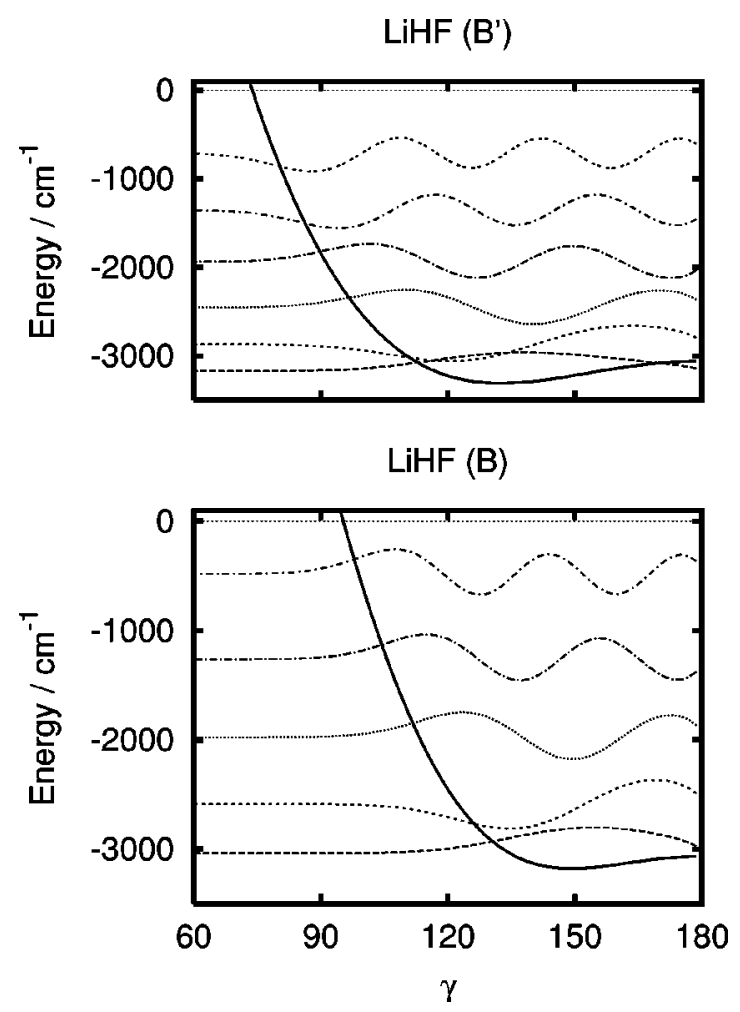

FIG. 8. Potential curves for $R=R_{e}$ and $r=r_{e}$ for the $B$ and $B^{\prime}$ states as a function of the angle and their first eigenvectors (placed at the eigenvalue). 
bound states become resonances. This fact will be discussed in more detail in the following.

In order to simulate the spectrum the line strength for an absorption from $\Psi_{k}^{J M \epsilon \alpha}$ eigenfunction in the $X$ state to $\Psi_{k^{\prime}}^{J^{\prime} M^{\prime} \epsilon^{\prime} \alpha^{\prime}}$ in the $A, B$, or $B^{\prime}$ states for an electric dipole transition is given by

$$
\begin{aligned}
S_{J \epsilon k \alpha}^{J^{\prime} \epsilon^{\prime} k^{\prime} \alpha^{\prime}}= & \left(\frac{1-\epsilon \epsilon^{\prime} \sigma \sigma^{\prime}}{2}\right)^{2} \frac{2 J+1}{3} \\
& \times \mid \sum_{v s j \Omega} \sum_{v^{\prime} s^{\prime} j^{\prime} \Omega^{\prime}} A_{v s j \Omega^{J \alpha k} A_{v^{\prime} s^{\prime} j^{\prime} \Omega^{\prime}}^{J^{\prime} \epsilon^{\prime} \alpha^{\prime} k^{\prime}}(-1)^{\Omega} A_{\Omega \Omega^{\prime}}} \\
& \times\left.\left(\begin{array}{ccc}
J & 1 & J^{\prime} \\
-\Omega & \Omega-\Omega^{\prime} & \Omega^{\prime}
\end{array}\right) M_{q, \alpha \alpha^{\prime}}^{v s j \Omega, v^{\prime} s^{\prime} j^{\prime} \Omega^{\prime}}\right|^{2},
\end{aligned}
$$

where the $A_{v s j \Omega}^{J \epsilon \alpha k}$ are the coefficients of the bound state in Eq. (11) and linearly polarized light has been assumed, so that $M=M^{\prime}$ and $S$ is independent of the particular $M$ value. In Eq. (12)

$A_{\Omega \Omega^{\prime}}=\left(1+\delta_{\Omega, 0}+\delta_{\Omega^{\prime}, 0}-\delta_{\Omega, 0} \delta_{\Omega^{\prime}, 0}\right) / \sqrt{\left(1+\delta_{\Omega, 0}\right)\left(1+\delta_{\Omega^{\prime}, 0}\right)}$, and the $M_{q, \alpha \alpha^{\prime}}^{v s j \Omega, v^{\prime} s^{\prime} j^{\prime} \Omega^{\prime}}$ matrix elements (with $q=\Omega-\Omega^{\prime}$ and $|q| \leqslant 1)$ are given by

$$
\begin{aligned}
M_{q, \alpha \alpha^{\prime}}^{v s j \Omega, v^{\prime} s^{\prime} j^{\prime} \Omega^{\prime}}= & \int d r \int d R \int d(\cos \gamma) \\
& \times Y_{j \Omega}^{*}(\gamma, 0) \varphi_{v}^{*}(r) \phi_{s}^{*}(R) d_{q}^{\alpha \alpha^{\prime}}(r, R, \gamma) \\
& \times Y_{j^{\prime} \Omega^{\prime}}(\gamma, 0) \varphi_{v}^{\prime}(r) \phi_{s}^{\prime}(R) .
\end{aligned}
$$

where $d_{q}^{X A}(r, R, \gamma)$ are the tensorial components of the transition electric dipole moments between the $X$ and $A, B, B^{\prime}$ electronic states referred to the body-fixed frame, obtained from those expressed in Cartesian coordinates in Eq. (5).

For low $J$, the projection of the total angular momentum on the $z$ body-fixed axis, $\Omega$, is a rather good quantum number. As an example, for $J=1$, there is always one dominant value of $\Omega$ with a weight larger than $99 \%$. As $J$ increases, the Coriolis coupling becomes more efficient and this clear assignment is no longer valid. For $J=10$ there is an important mixing, but there is always one $\Omega$ with a dominant contribution larger than $50 \%$. For these high $J$ the total number of basis set functions increases so much that variational methods cannot be used to diagonalize the Hamiltonian matrix. Instead, we use an iterative procedure based on the Lanczos algorithm ${ }^{70}$ in two steps. The eigenvalues are obtained with a non-orthogonal Lanczos procedure following the method of Cullum and Willoughby. ${ }^{71}$ The eigenstates are then obtained iteratively using the conjugate gradient method. ${ }^{72,73}$ This method is rather efficient for low excitation. However, it becomes very time consuming when states near the dissociation limits are required, as is the case here. Moreover, if resonances above this limit are reached, this method is no longer valid and some alternative methods should be used.

\section{B. Wave packet method and spectra simulations}

This method does not only allow one to calculate resonances, but also facilitates the calculation of highly excited bound states, which becomes very difficult to calculate using standard bound state methods. The wave packet method uses grids which can be increased as desired without having to store and diagonalize large matrices.

Using a first-order perturbation treatment, the total absorption cross section for the absorption from $\Psi_{k}^{J M \epsilon \alpha}$ eigenfunction in the $X$ state to $\Psi_{k^{\prime}}^{J^{\prime} M^{\prime} \epsilon^{\prime} \alpha^{\prime}}$ in the $A, B$, or $B^{\prime}$ states for an electric dipole transition is given by the Fourier transform of the autocorrelation function as

$$
\begin{aligned}
\sigma^{J^{\prime} \alpha^{\prime} \leftarrow J, k, \alpha}(\hbar \omega) \propto & \frac{1}{2 \pi \hbar} \int_{-\infty}^{\infty} d t e^{i E t / \hbar} \\
& \times\left\langle\Psi_{k \alpha}^{J^{\prime} \epsilon^{\prime} \alpha^{\prime}}(\mathbf{r}, \mathbf{R}, t=0) \mid \Psi_{k \alpha}^{J^{\prime} \epsilon^{\prime} \alpha^{\prime}}(\mathbf{r}, \mathbf{R}, t)\right\rangle,
\end{aligned}
$$

with $\hbar \omega=E-E_{i}$ and where the initial wave packet is defined as ${ }^{52}$

$$
\begin{aligned}
\Psi_{k \alpha}^{J^{\prime} \epsilon^{\prime} \alpha^{\prime}}(\mathbf{r}, \mathbf{R}, t=0)= & \sum_{\Omega^{\prime}} W_{M^{\prime} \Omega^{\prime}}^{J^{\prime} \epsilon^{\prime} \alpha^{\prime}}(\phi, \theta, \chi) \\
& \times\left\langle W_{M^{\prime} \Omega^{\prime}}^{J^{\prime} \epsilon^{\prime} \alpha^{\prime}}|\mathbf{d} \cdot \mathbf{e}| \Psi_{k}^{J M \epsilon^{\prime} \alpha}\right\rangle,
\end{aligned}
$$

where $\mathbf{e}$ is the polarization vector of the incident photon and d is the electric dipole transition moment for the desired electronic transitions.

The price to pay using wave packet methods is that the propagation should be performed up to very long times, infinity in principle, to resolve completely the structure dealing with bound states. To overcome this difficulty, each transition will be "dressed" by a Lorentzian function, characterized by a half-width at half-maximum $\Gamma$, as done previously for $\mathrm{Na}-\mathrm{HF}^{74}$ and $\mathrm{Li}-\mathrm{HF}^{75}$ The width corresponds to electronic predissociation broadening and, also, would simulate the rotational broadening of the spectrum. This last broadening must be considered because of the relatively high rotational temperature of the $\mathrm{Li}-\mathrm{HF}$ complex obtained experimentally in the cross beams. ${ }^{25,26}$ In the present treatment the inclusion of the width is performed by multiplying the autocorrelation function by a decaying exponential such that the expression of the spectrum associated with a single transition, in Eq. (14), transforms to

$$
\begin{aligned}
\sigma_{\Gamma}^{J^{\prime} \alpha^{\prime} \leftarrow J, k, \alpha}(\hbar \omega) \propto & \frac{1}{2 \pi \hbar} \int_{-\infty}^{\infty} d t e^{i E t / \hbar} e^{-\Gamma t / \hbar} \\
& \times\left\langle\Psi_{k \alpha}^{J^{\prime} \epsilon^{\prime} \alpha^{\prime}}(\mathbf{r}, \mathbf{R}, t=0) \mid \Psi_{k \alpha}^{J^{\prime} \epsilon^{\prime} \alpha^{\prime}}(\mathbf{r}, \mathbf{R}, t)\right\rangle,
\end{aligned}
$$

which allows one to stop the propagation at shorter times. In this work the maximum time considered has been 20 ps for $J<3$ and 5 ps for higher $J$ 's. The details of the calculation can be found elsewhere. ${ }^{41,48,52}$ Here convergence was obtained with a radial grid in $r, R$ composed by $32 \times 128$, with $0.45 \leqslant r \leqslant 2 \AA$ and $0.5 \leqslant R \leqslant 12 \AA$, and 30 Gauss-Legendre points for the angle $\gamma$. Finally, all possible $\Omega$ projections are included in the calculations.

To interpret the absorption spectrum we start with the simplest transitions: $A, B$ and $B^{\prime} \quad J=0 \leftarrow X, J=1$. For the cases of $A$ and $B$, the transitions considered 


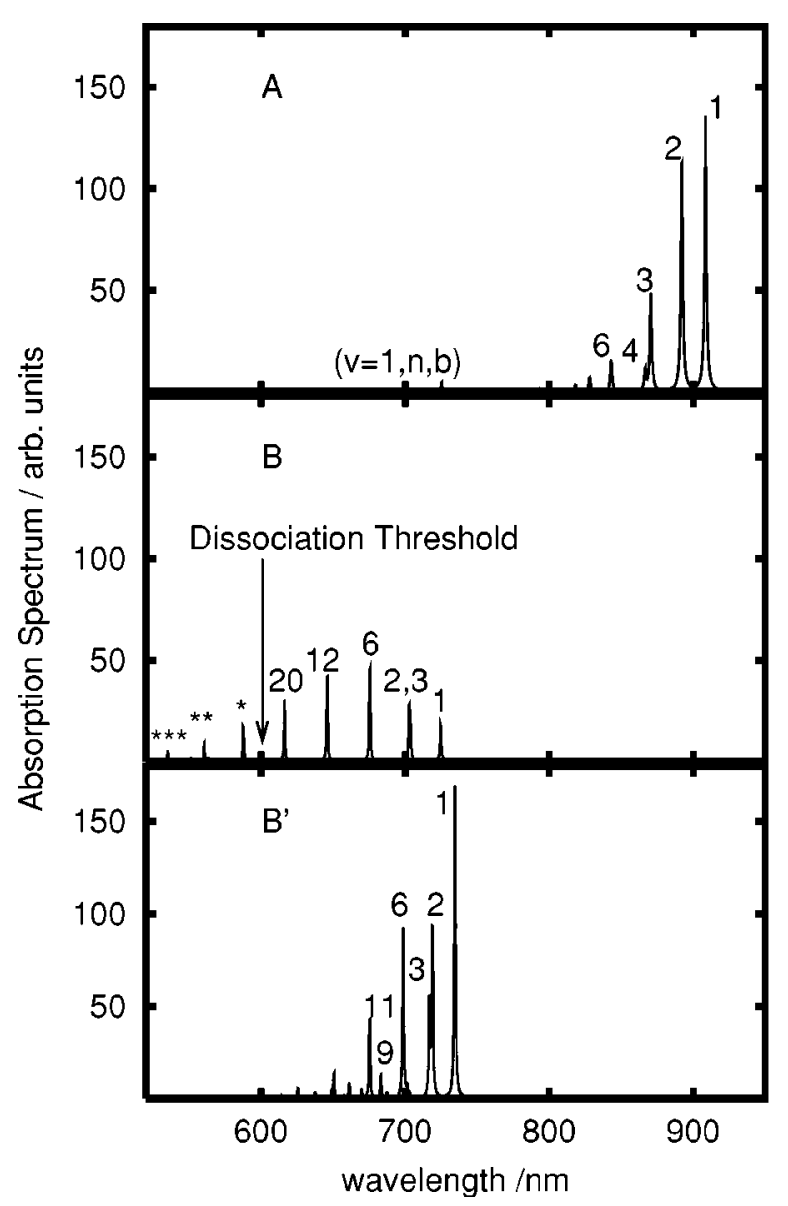

(a)

(b)

(c)

FIG. 9. Absorption spectra for the (a) $A, J=0\left(v, n, b, \Omega^{\epsilon}\right)=(v, n, b, \Omega$ $\left.=0^{+}\right) \leftarrow X, J=1\left(0,0,0,0^{-}\right), \quad$ (b) $\quad B, J=0\left(v, n, b, \Omega^{\epsilon}\right)=\left(v, n, b, \Omega=0^{+}\right)$ $\leftarrow X, J=1\left(0,0,0,0^{-}\right)$, and (c) $B^{\prime}, J=0\left(v, n, b, \Omega=0^{-}\right) \leftarrow X, J=1\left(0,0,0,1^{+}\right)$ transitions simulated using Eq. (12) as a function of wavelength. The labels of the well correspond to the $n_{\text {vib }}$ ordering quantum number used in Table IV, where the assignment can be found (see also Fig. 7).

correspond to $\quad A, B, J=0\left(v, n, b, \Omega^{\epsilon}\right)=\left(v, n, b, \Omega=0^{+}\right)$ $\leftarrow X, J=1\left(0,0,0,0^{-}\right)$. For $B^{\prime}$ state, because of its $A^{\prime \prime}$ symmetry the total parity changes, and the transitions are $B^{\prime}, J$ $=0\left(v, n, b, \Omega=0^{-}\right) \leftarrow X, J=1\left(0,0,0,1^{+}\right)$. The peaks appearing in the spectrum, in Fig. 9 are labeled with the ordering quantum number. After inspection of Table IV it can be seen that in nearly all the cases, the peaks correspond to different bending excitation levels for the three final excited electronic states. In the rest of the cases, the levels are pretty close to excited bending levels. In fact in such situations, there is an interaction between pure zero-order stretching and bending levels which give rise to "mixed" states. Therefore, the oscillator strength is borrowed by the pure bending zero-order component. This result can be understood by the topology of the different PESs near the wells, as discussed earlier. The equilibrium distances are nearly the same in the four cases, the major difference being the equilibrium angle. As a consequence, since the dipole transition moment does not strongly vary in the Frank-Condon region, only pure bending excitation states present appreciable overlap with the ground initial state.

It should be noted that the agreement between bound state and wave-packet calculations is excellent up to

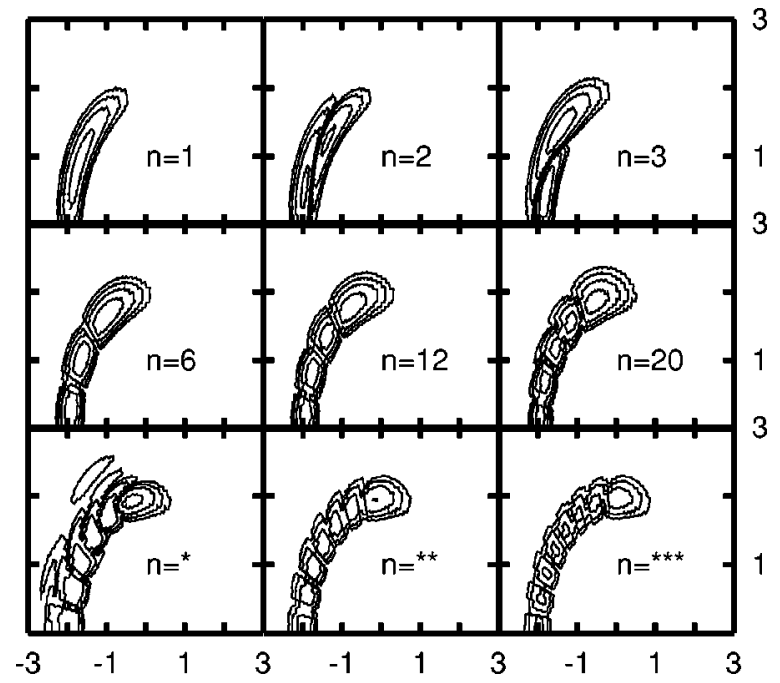

FIG. 10. Contour plots of the probability density associated to the bound states and resonances obtained using the wave-packet method for $\mathrm{Li}-\mathrm{HF}$ $(B, J=0)$. Each number corresponds to those used in Fig. 9 to label the peaks of the spectrum. Distances in $\AA$.

$\mathrm{Li}\left({ }^{2} P\right)+\mathrm{HF}(v=0)$ dissociation limit. The peaks appearing in the $B$ state above this dissociation limit, correspond to resonances and do not appear in the bound state calculation. For this reason in the theoretical spectrum obtained using bound state calculations presented in the following there is no appreciable absorption intensity below $600 \mathrm{~nm}$ while the experimental one still shows several relevant peaks.

In order to assign those peaks, the bound components $\phi_{n}^{(0)}$ of the associated resonances is approximately extracted from the wave packet calculations ${ }^{76,77,52}$ as

$$
\phi_{n}^{(0)} \approx \mathcal{R} \int_{0}^{\infty} d t e^{i\left(E_{n}+i \Gamma_{n}\right) t / \hbar} \Psi_{k \alpha}^{J^{\prime} \epsilon^{\prime} \alpha^{\prime}}(\mathbf{r}, \mathbf{R}, t),
$$

where $E_{n}$ and $\Gamma_{n}$ are the approximate position and width of each resonance, respectively, and here $\Gamma_{n}$ is taken as $1 \mathrm{~cm}^{-1}$ for all of them. In Fig. 10 the contour plot of the probability density of each resonance labeled in Fig. 9 is shown. For those peaks below the first dissociation limit, the structure is in perfect agreement with that of the bound state calculations in Fig. 7, and correspond to a bending progression with no stretching excitation, either in $r$ or $R$, as discussed earlier. For energies above the $\operatorname{Li}\left({ }^{2} P\right)+\operatorname{HF}(v=0)$ threshold, this bending progression is continued, so that also those peaks below $600 \mathrm{~nm}$ can be assigned. Moreover, such net separation between the bending and stretching modes justifies why those resonances are so long-lived: after more than 20 ps of propagation there is no significant fragmentation toward the $\mathrm{Li}\left({ }^{2} P\right)+\mathrm{HF}(v=0)$ channel.

Because the simple $J=0 \leftarrow J=1$ rotational transition considered in Fig. 9, for the case of $A$ and $B$ electronic states, only bound states with $\Omega=0$ are considered, i.e., only parallel transitions are included. When higher angular momentum are included, these transitions split in two, one parallel and the other perpendicular. For the $B^{\prime} \leftarrow X$, however, only the perpendicular transitions are possible. As a consequence, several rotational transitions must be included to better simulate the spectrum. Moreover, in Fig. 9 only the 
a) Using Bound states calculations

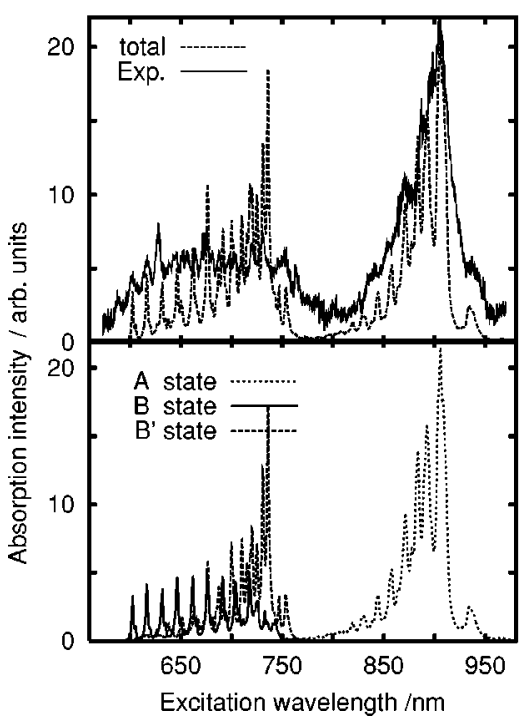

b) Using wavepacket calculations

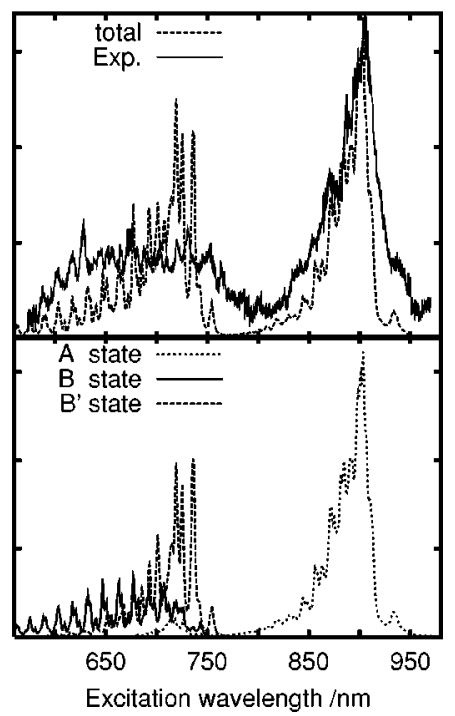

FIG. 11. Absorption spectra for the (a) $A, B$ or $B^{\prime}, J^{\prime}$ $=0,1,2,3 \leftarrow X, J=0,1,2$ transitions simulated using Eq. (12) and (b) $A, B$ or $B^{\prime}, J^{\prime}=9,10,11 \leftarrow X, J=10, \epsilon=-1$ transitions simulated using the wave packet method. A vibrational temperature of $250 \mathrm{~K}$. Each transition has been dressed with a Lorentzian with a width of $10 \mathrm{~cm}^{-1}$. In the top panels the simulated total spectra are compared with the experimental result of Ref. 26. In the bottom panels the individual contribution to the three final electronic states is presented. ground vibrational state is considered. However, the experimental spectrum of Hudson et al. ${ }^{26}$ is obtained at high vibrational and rotational temperatures. Therefore, to better simulate the spectrum transitions from different vibrational states of the $X$ state should be included, assuming a Boltzman distribution. These two facts are included in the simulated spectra presented in Fig. 11. In Fig. 11(a), all transitions from $X$, $J=0,1$, and 2 to $A, B, B^{\prime} J^{\prime}=0,1,2$, and 3 are considered using Eq. (12), for which bound states have been calculated to energies close to the dissociation energy for each of the four electronic states. In Fig. 11(b), the transitions from $X, J=10, \epsilon=-1, n=1,2, \ldots, 10$ to $A, B, B^{\prime}, J=9,10$, and 11 , that involve 30 different wave packet calculations are reported. The peaks of the $A$ band (above $850 \mathrm{~nm}$ ) are pretty well reproduced, and also those appearing below $700 \mathrm{~nm}$, which essentially correspond to the $B$ state. The intermediate region, between 700 and $850 \mathrm{~nm}$, is the worst reproduced, having contributions from the three electronic states. In particular, the intensity is too high about $740 \mathrm{~nm}$ and too low between 750 and $800 \mathrm{~nm}$. The intensity at $740 \mathrm{~nm}$ decreases with increasing $J$, while the contribution of the $A$ state about this wavelength increases.

At the high temperatures obtained in the experimental spectrum, $\approx 200-300 \mathrm{~K}$, pretty high total angular momentum (up to $J=30$ or more) must be included, and also high vibrational states of the initial $\mathrm{Li}-\mathrm{HF}(X)$ complex. It is then interesting to address the question of whether or not the intensity discrepancies between 750 and $800 \mathrm{~nm}$ can be solved by properly accounting for the average over the initial state distribution. "Exact" calculations of the bound states at high total angular momentum are feasible but it is very difficult to converge eigenstates close to the dissociation threshold. On the other side, the wave packet method would require a large number of independent calculations. As an alternative, approximated bound calculations have been performed neglecting the Coriolis coupling term (centrifugal sudden approximation) so that for a given $J, 2 J+1$ independent calculations have to be performed for each $\Omega$ projection, individually. In this approach, bound state calculations have been performed for $J=0,1,2, \ldots, 30$ for the four electronic states and for $\Omega=0,1, \ldots, 7$. Individual transitions, calculated using Eq. (12), are "dressed" with a Lorentzian of variable width. The average of different temperature of the complexes is performed assuming a Boltzmann distribution. Such simulations are presented in Fig. 12 for several temperatures and widths. As in the previous case, increasing the temperature the intensity spreads and around $730 \mathrm{~nm}$ it decreases, improving the agreement with the experimental results. However, the effect does not seem to be enough.

\section{Nonadiabatic effects and interpretation of the spectrum}

There are several reasons which could explain this disagreement. First of all, the transition dipole moment could be too large between the $X$ and $B^{\prime}$ states. However, several results at different levels of calculations and basis sets indicates that our results are rather insensitive to those factors.

Second, the $a b$ initio energies and the global fits. In particular that of the $B^{\prime}$ state. Previous calculations by Jasper et $a l .^{42}$ reported $a b$ initio points for the $B^{\prime}$ state very close to those of the $A$ state. If so, the band corresponding to the $B^{\prime}$ state would shift toward larger wavelengths, but probably too much. However, in the present calculations it was not possible to obtain such results, as discussed earlier. Also, it could be the slope of the PESs around the Frank-Condon region. In this direction, detailed $a b$ initio calculations have been performed in this region and several parametrization of the fits, obtaining pretty similar results to those reported here.

A third reason could be the nonadiabatic couplings among the four electronic states. In the simulated spectra a single width has been assumed for all the states. However, preliminary calculations ${ }^{78}$ using a time-dependent GoldenRule approach ${ }^{79}$ in an adiabatic representation for which nonadiabatic couplings have been calculated, indicate that 


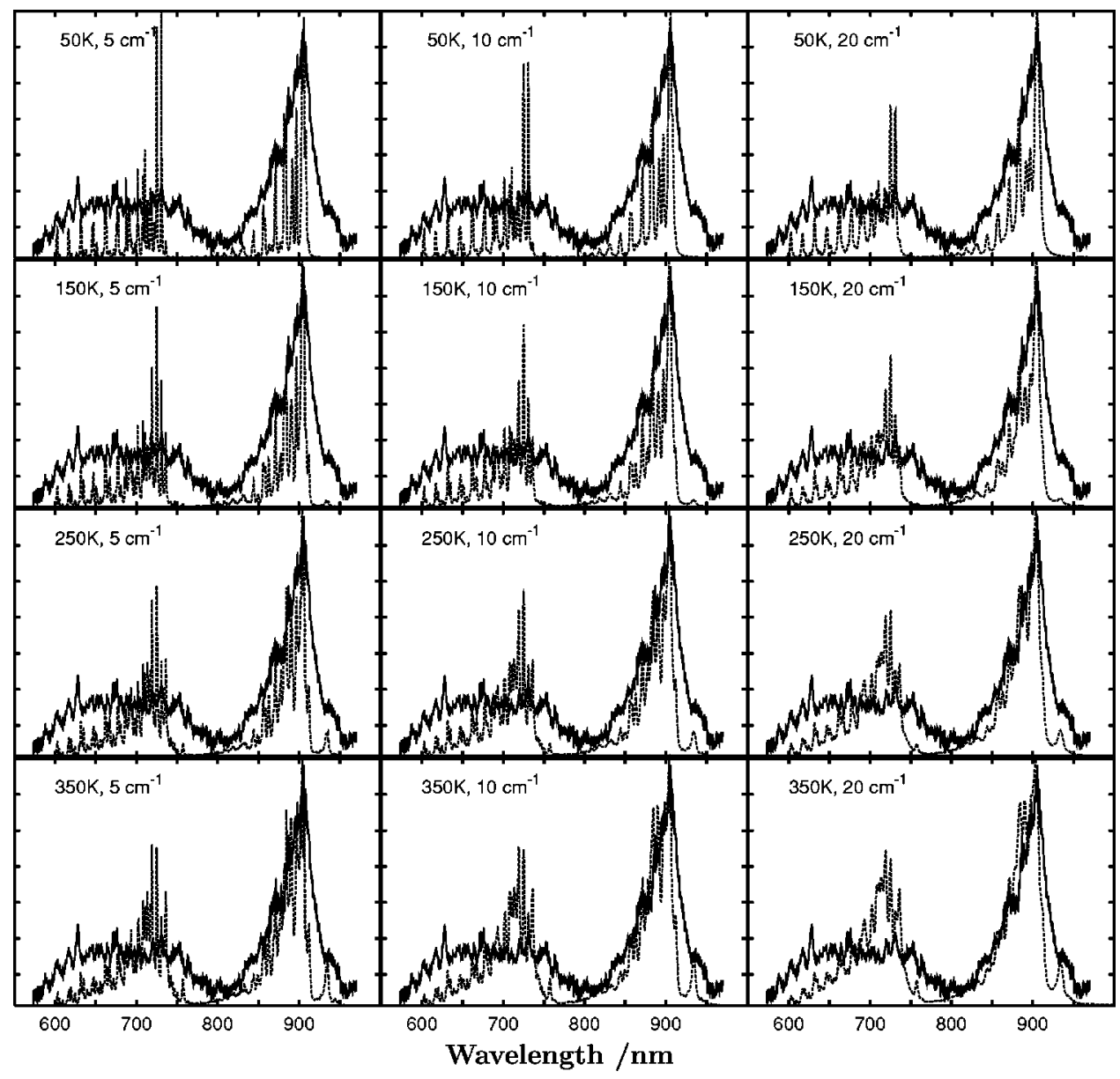

FIG. 12. Simulated absorption spectra, in solid, as a function of the width and vibrational temperature obtained using the bound state method in the centrifugal sudden approach, calculating all bound states from $J=0$ to $J=30$, and from $\Omega=0$ to $\Omega=7$ for the $X, A, B$, and $B^{\prime}$ electronic states. In dashed lines is the experimental spectrum from Ref. 26.

the electronic predissociation (EP) rates are very much dependent on the vibrational and mainly on the electronic state. The rates for the $A$ state are rather large, of the order of few wave numbers, one order of magnitude larger than the semiclassical estimate by Jasper et al. ${ }^{54}$ in which a quasidiabatic $X-A$ model potential is used without calculating nonadiabatic couplings. The situation of the $B$ and $B^{\prime}$ states is rather different, because their couplings with the ground electronic state is either negligible or vanish. However, there is no indication of such situation, and the widths of the vibrational levels of all the electronic states should be of similar order.

In fact, it should be taken into account that between 600 and $750 \mathrm{~nm}$ there is a high density of bound states of the three excited electronic states (see Fig. 11). There are several crossings between the $A$ and $B$ states, Fig. 4, which suggests the appearance of strong couplings between them, especially as a function of the angle. In this situation, the rovibrational levels of different electronic states will interact among them, giving rise to a broadening which may somehow distribute the absorption intensity according to the experiment. As stated earlier, a crude simple model in which $R$ and $r$ are fixed to their equilibrium values leading to one effective internal coordinate, $\gamma$, is able to reproduce the main features of the spectrum. Here, for $r_{e}=1.9$ a.u. and $R_{e}=3.41$ a.u., the angular nonadiabatic coupling between the $A$ and $B$ states has been calculated using the finite difference method of Ref. 80. These two states have been diabatized using the method of Simah, Hartke, and Werner, ${ }^{81}$ giving rise to two new elec- tronic states, labeled as $|Z\rangle$ and $|X\rangle$, in which the $p$ orbital of the $\mathrm{Li}$ atom has a fixed orientation with respect to the $\mathrm{HF}$ axes. The $B^{\prime}$ (or equivalently $|Y\rangle$ ) state is not directly coupled to $|Z\rangle$ and $|X\rangle$ by symmetry. However, when taking into account the nuclear motions such condition relax, because there are rotational functions with the two parities under total inversion of spatial coordinates, which for the electronic variables in the body-fixed frame transform in the reflection through the $x z$ plane. In order to fully describe this coupling it is convenient to transform them to the electronic states $|\Lambda\rangle$ with well-defined projection of the electronic orbital angular momentum, $\Lambda$, through the well-known expression

$$
\left(\begin{array}{c}
|Z\rangle \\
|X\rangle \\
|Y\rangle
\end{array}\right)=\left(\begin{array}{ccc}
1 & 0 & 0 \\
0 & -1 / \sqrt{2} & 1 / \sqrt{2} \\
0 & i / \sqrt{2} & i / \sqrt{2}
\end{array}\right)\left(\begin{array}{c}
|0\rangle \\
|+1\rangle \\
|-1\rangle
\end{array}\right) .
$$

In this new representation the $| \pm 1\rangle$ states are degenerate and cross with the $|0\rangle$ state, as can be seen in Fig. 13.

Within the reduced dimensionality approach, the bound states are expanded as

$\Psi_{k}(\hat{r}, \hat{R})=\sqrt{\frac{2 J+1}{8 \Pi^{2}}} \sum_{j \Omega \Lambda} C_{j \Omega \Lambda}^{k} D_{M \Omega}^{J *}(\phi, \theta, \chi) Y_{j, \Omega-\Lambda}(\gamma)|\Lambda\rangle$,

and calculations from $J=0$ to 30 have been performed, neglecting the Coriolis couplings. Zero-point energy correc- 


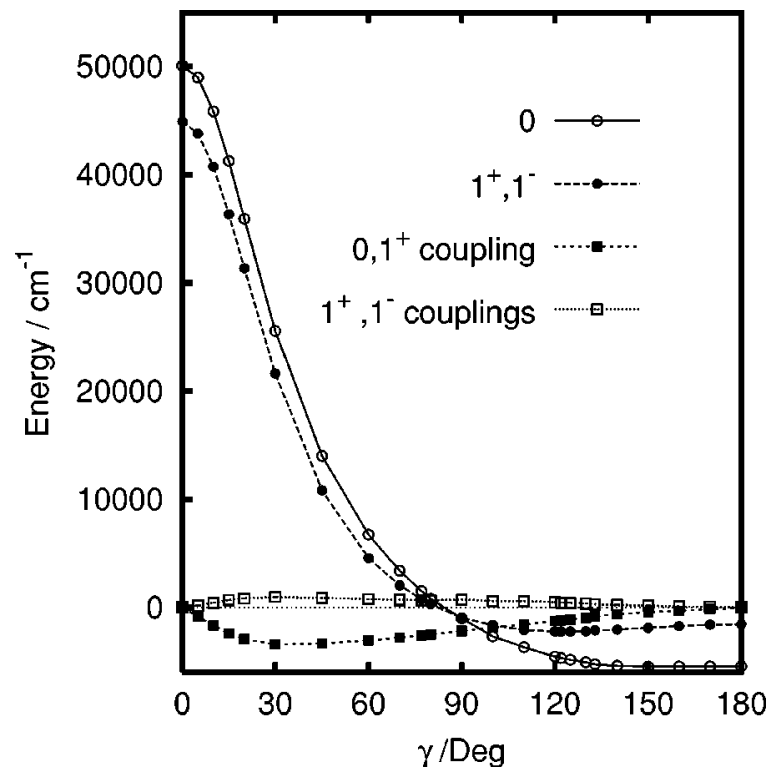

FIG. 13. Potential energy curves, and couplings, of the excited electronic states in the $\Lambda$ diabatic basis sets for $r=1.9$ and $R=3.41$ a.u.

tions were introduced by shifting the diagonal matrix elements of the Hamiltonian matrix by a value estimated comparing to the full calculations in the adiabatic representation. Individual line strengths were obtained as explained before, "dressing" each line with a Lorentzian function of $20 \mathrm{~cm}^{-1}$, and the spectrum was obtained after averaging over an initial Boltzman distribution at $250 \mathrm{~K}$. The resulting spectrum, in Fig. 14, is compared with that obtained in the

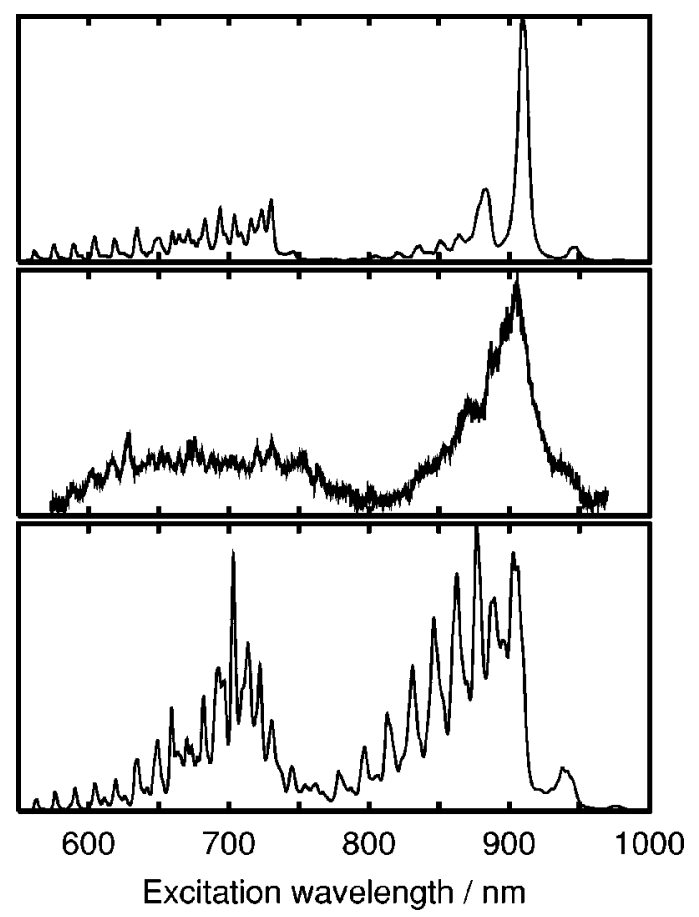

FIG. 14. Absorption spectrum obtained using the crude monodimensional model in a diabatic basis set (top panel), compared with that obtained in the adiabatic representation (bottom line) neglecting nonadiabatic couplings, with $\Gamma=20 \mathrm{~cm}^{-1}$ and $250 \mathrm{~K}$. Also the experimental spectrum of Hudson et al. (Ref. 26) is shown (middle panel). adiabatic representation to analyze the effect of nonadiabatic couplings, and with the experimental one. It can be observed, first, that this simple model is able to reproduce quite reasonably the main features of the spectrum, confirming that the bands can be assigned to bending progressions. Second, in the diabatic representation the relative intensity of the two bands of the spectrum is better represented, confirming the hypothesis that to get a better agreement with the experimental results nonadiabatic couplings should be taken into account. Of course, the frozen degrees of freedom are important to properly describe the density of states. As an example, the density of states in between the two bands is not properly described. Also, the first stretching excitation in the $A$ state cannot be obtained in this model and, as a consequence, some of the peaks near the maximum of the $A$ band are not present. However, the model is rather satisfactory, especially when its simplicity is considered. A more detailed study about this and the electronic predissociation nonadiabatic phenomena is now in progress. ${ }^{78}$

It is interesting to note, in the experimental spectrum below $650 \mathrm{~nm}$, the fact that the spacing between the peaks increases with decreasing wavelength is surprising and it was first attributed to different initial vibrational states of the $\operatorname{LiHF}(X)$ complex. In the present study, however, the assignment is different, being attributed to a bending progression of the $B^{\prime}$ state, as found before. ${ }^{45}$ Even more, the sequences of peaks appearing in the spectrum can also be attributed to be mainly due to bending excitation in the different electronic states providing a very clear and simplified assignment.

\section{CONCLUSIONS}

A new atomic basis set has been determined for $\mathrm{Li}$ and $\mathrm{H}$ atoms which yields rather good atomic excitation energies as well as quite precise diatomic potential curves in several electronic states. In order to study the LiHF triatomic system, about $6000 a b$ initio points have been calculated in the $A$ and $A^{\prime}$ representation of the $C_{s}$ point group. Four new global PESs, corresponding to the first four electronic states, have been individually fitted, with rather low rms error. The three excited surfaces have been shifted by $50 \mathrm{~cm}^{-1}$ to reproduce the experimental $\operatorname{Li}\left({ }^{2} P \leftarrow{ }^{2} S\right)$ excitation energy. Because of this shift, added to some other errors commented on above, the PES of the $A$ state was corrected by a multiplying parameter which was introduced to better reproduce the experimental spectrum. ${ }^{26}$ This parameter together with the experimental $\operatorname{Li}\left({ }^{2} P \leftarrow{ }^{2} S\right)$ excitation energy were the only non $a b$ initio parameters introduced in the PESs presented here.

With these new PESs, the $A, B, B^{\prime} \leftarrow X$ absorption spectrum of the LiHF has been simulated, and the results are in very good agreement with the experimental data of Hudson et al. ${ }^{26}$ Very excited rovibrational states, near the first dissociation energy (below and above), were needed to simulate the spectrum. Two different methods have been used, one based in bound states and the second in wave packet calculations. Each of the methods present its own advantages and problems. The simulated spectra present a very good agreement with the experimental one ${ }^{26}$ and several alternatives have been used to simulate the relatively high temperatures at which the complex was experimentally obtained. 
The structure of the spectrum was interpreted to be essentially a bending progression, with nearly no excitation of the stretching modes. Moreover, the bending energy separation of the $B$ and $B^{\prime}$ states increases with increasing energy, due to the shape of the potential as a function of the internal angle. This fact has been nicely reproduced by a simple onedimensional model. Also, because of this unusual energy separation, the peaks at short wavelengths appearing in the experimental spectrum were first thought to be due to transitions from different vibrational states of LiHF in the ground electronic state. The simulation presented here, however, allows one to interpret and assign all those peaks to transitions to bending progressions in the excited electronic states, which energy spacings increase in the $B$ and $B^{\prime}$ cases. The disagreement in the relative intensities of the bands of the spectrum has been attributed to nonadiabatic effects which are now being studied. ${ }^{78}$

It would be desirable to get some more experimental data at lower temperatures to be able to separate the individual transitions and measure the lifetime of the individual excited states. Also, we are currently studying the electronic predissociation from the excited electronic states to determine not only the lifetimes as a function of the excitation of the different vibrational modes, but also to determine the branching ratio between the two rearrangement channel, $\mathrm{HF}+\mathrm{Li}$ and $\mathrm{LiF}+\mathrm{H}$, in the ground electronic state. This information is expected to be crucial to understanding the role of nonadiabatic couplings in the reaction dynamics. Also, the introduction of these nonadiabatic couplings in the simulation of reactive scattering is expected to explain, at least partially, the disagreement between theory and recent experiments performed by Loesch and co-workers. ${ }^{38}$

\section{ACKNOWLEDGMENTS}

This work has been supported by MCYT (Spain), under Grant Nos. BQU2001-0152 and BFM2001-2179. C.S. wants to acknowledge an I3P grant. Also we want to thank Professor J.C. Polanyi for providing us with the numerical data of the experimental spectrum and Dr. F. Naumkin for very interesting and fruitful discussion.

${ }^{1}$ J. L. Magee, J. Chem. Phys. 8, 687 (1940).

${ }^{2}$ R. D. Levine and R. B. Bernstein, Molecular Reaction Dynamics and Chemical Reactivity (Oxford University Press, New York, 1987).

${ }^{3}$ E. H. Taylor and S. Datz, J. Chem. Phys. 23, 1711 (1955).

${ }^{4}$ D. R. Herschbach, Adv. Chem. Phys. 10, 319 (1966).

${ }^{5}$ P. S. Weiss, J. M. Mestdagh, M. H. Covinsky, B. A. Balko, and Y. T. Lee, Chem. Phys. 126, 93 (1988).

${ }^{6}$ R. Düren, U. Lackschewitz, and S. Milosevic, Chem. Phys. 126, 81 (1988).

${ }^{7}$ T. J. Odiorne, P. R. Brooks, and J. V. V. Kasper, J. Chem. Phys. 55, 1980 (1971).

${ }^{8}$ J. G. Pruett and R. N. Zare, J. Chem. Phys. 64, 1774 (1976).

${ }^{9}$ Z. Karny, R. C. Estler, and R. N. Zare, J. Chem. Phys. 69, 5199 (1978).

${ }^{10}$ Z. Karny and R. N. Zare, J. Chem. Phys. 68, 3360 (1978).

${ }^{11}$ F. E. Bartoszek, B. A. Blackwell, J. C. Polanyi, and J. J. Sloan, J. Chem. Phys. 74, 3400 (1981).

${ }^{12}$ R. Zhang, D. J. Rakestraw, K. G. McKendrick, and R. N. Zare, J. Chem. Phys. 89, 6283 (1988).

${ }^{13}$ M. Hoffmeister, R. Schleysing, F. Stienkemeier, and H. J. Loesch, J. Chem. Phys. 90, 3528 (1989).

${ }^{14}$ B. A. Blackwell, J. C. Polanyi, and J. J. Sloan, Chem. Phys. 30, 299 (1978).
${ }^{15}$ C.-K. Man and R. C. Estler, J. Chem. Phys. 75, 2779 (1981).

${ }^{16}$ H. H. Dispert, M. W. Geis, and P. R. Brooks, J. Chem. Phys. 70, 5317 (1979).

${ }^{17}$ M. Hoffmeister, L. Potthast, and H. J. Loesch, Chem. Phys. 78, 369 (1983).

${ }^{18}$ C. Jouvet, M. Boivineau, M. C. Duval, and B. Soep, J. Phys. Chem. 91, 5416 (1987).

${ }^{19}$ B. Soep, C. J. Whitham, A. Keller, and J. P. Visticot, Faraday Discuss. Chem. Soc. 91, 191 (1991).

${ }^{20}$ B. Soep, S. Abbés, A. Keller, and J. P. Visticot, J. Chem. Phys. 96, 440 (1992).

${ }^{21}$ A. Keller, R. Lawruszczuk, B. Soep, and J. P. Visticot, J. Chem. Phys. 105, 4556 (1996).

${ }^{22}$ R. Lawruszczuk, M. Elhanine, and B. Soep, J. Chem. Phys. 108, 8374 (1998).

${ }^{23}$ K. Liu, J. C. Polanyi, and S. Yang, J. Chem. Phys. 98, 5431 (1993).

${ }^{24}$ J. C. Polanyi and J.-X. Wang, J. Phys. Chem. 99, 13691 (1995).

${ }^{25}$ X. Y. Chang, R. Ehlich, A. J. Hudson, P. Piecuch, and J. C. Polanyi, Faraday Discuss. 108, 411 (1997).

${ }^{26}$ A. J. Hudson, H. B. Oh, J. C. Polanyi, and P. Piecuch, J. Chem. Phys. 113, 9897 (2000)

${ }^{27}$ A. J. Hudson, F. Y. Naumkin, H. B. Oh, J. C. Polanyi, and S. Raspopov, Faraday Discuss. (in press).

${ }^{28}$ S. Skowronek, R. Pereira, and A. González-Ureña, J. Chem. Phys. 107, 1668 (1997)

${ }^{29}$ S. Skowronek, R. Pereira, and A. González-Ureña, J. Phys. Chem. A 101, 7468 (1997).

${ }^{30}$ S. Skowronek, J. B. Jiménez, and A. González-Ureña, Chem. Phys. Lett. 303, 275 (1999).

${ }^{31}$ V. Stert, P. Farmanara, W. Radloff, F. Noack, S. Skowronek, J. B. Jimenez, and A. González-Ureña, Phys. Rev. A 59, R1727 (1999).

${ }^{32}$ P. Farnamara, V. Stert, W. Radloff, S. Skowronek, and A. González-Ureña, Chem. Phys. Lett. 304, 127 (1999).

${ }^{33}$ C. H. Becker, P. Casavecchia, P. W. Tiedemann, J. J. Valentini, and Y. T. Lee, J. Chem. Phys. 73, 2833 (1980).

${ }^{34}$ H. J. Loesch, E. Stenzel, and B. Wüstenbecker, J. Chem. Phys. 95, 3841 (1991).

${ }^{35}$ H. J. Loesch and F. Stienkemeier, J. Chem. Phys. 98, 9570 (1993).

${ }^{36}$ H. J. Loesch and F. Stienkemeier, J. Chem. Phys. 99, 9598 (1993).

${ }^{37}$ F. J. Aoiz, E. Verdasco, V. Sáez-Rabanos, H. J. Loesch, M. Menéndez, and F. Stienkemeier, Phys. Chem. Chem. Phys. 2, 541 (2000).

${ }^{38}$ O. Höbel, M. Menéndez, and H. J. Loesch, Phys. Chem. Chem. Phys. 3, 3633 (2001).

${ }^{39}$ G. A. Parker, A. Laganà, S. Crocchianti, and R. T Pack, J. Chem. Phys. 102, 1238 (1995).

${ }^{40}$ A. Aguado, M. Paniagua, M. Lara, and O. Roncero, J. Chem. Phys. 106, 1013 (1997).

${ }^{41}$ A. Aguado, M. Paniagua, M. Lara, and O. Roncero, J. Chem. Phys. 107, 10085 (1997).

${ }^{42}$ A. W. Jasper, M. D. Hack, D. G. Truhlar, and P. Piecuch, J. Chem. Phys. 116, 8353 (2002).

${ }^{43}$ A. Aguado, M. Paniagua, and H.-J. Werner (unpublished).

${ }^{44}$ A. Aguado, M. Lara, M. Paniagua, and O. Roncero, J. Chem. Phys. 114, 3440 (2001).

${ }^{45}$ C. Sanz, O. Roncero, M. Paniagua, and A. Aguado, Chem. Phys. Lett. 351, 295 (2002).

${ }^{46}$ M. Baer, I. Last, and H. J. Loesch, J. Chem. Phys. 101, 9648 (1994).

${ }^{47}$ F. Gögtas, G. G. Balint-Kurti, and A. R. Offer, J. Chem. Phys. 104, 7927 (1996).

${ }^{48}$ M. Lara, A. Aguado, O. Roncero, and M. Paniagua, J. Chem. Phys. 109, 9391 (1998).

${ }^{49}$ M. Lara, A. Aguado, M. Paniagua, and O. Roncero, J. Chem. Phys. 113, 1781 (2000)

${ }^{50}$ F. J. Aoiz, M. T. Martínez, M. Menéndez, V. Sáez Rábanos, and E. Verdasco, Chem. Phys. Lett. 299, 25 (1999).

${ }^{51}$ M. Paniagua, A. Aguado, M. Lara, and O. Roncero, J. Chem. Phys. 109, 2971 (1998).

${ }^{52}$ M. Paniagua, A. Aguado, M. Lara, and O. Roncero, J. Chem. Phys. 111, 6712 (1999).

${ }^{53}$ R. Burcl, P. Piecuch, V. Spirko, and O. Bludský, J. Mol. Struct. 591, 151 (2002).

${ }^{54}$ A. W. Jasper, M. D. Hack, A. Chakraborty, D. G. Truhlar, and P. Piecuch, J. Chem. Phys. 116, 8353 (2002).

${ }^{55}$ Y. Zeiri and M. Shapiro, Chem. Phys. 31, 217 (1978). 
${ }^{56}$ M. Shapiro and Y. Zeiri, J. Chem. Phys. 70, 5264 (1979).

${ }^{57}$ T. H. Dunning, Jr., J. Chem. Phys. 90, 1007 (1989).

${ }^{58}$ R. A. Kendall, T. H. Dunning, Jr., and R. J. Harrison, J. Chem. Phys. 96, 6796 (1992)

${ }^{59}$ D. R. Yarkony, Int. J. Quantum Chem. 31, 91 (1987).

${ }^{60}$ A. Boutalib and F. X. Gadéa, J. Chem. Phys. 97, 1144 (1992).

${ }^{61}$ MOLPRO is a package of $a b$ initio programs designed by H.-J. Werner and P. J. Knowles, with contributions from J. Almlöf, R. D. Amos, A. Berning et al. (version 2002.7).

${ }^{62}$ H.-J. Werner and P. J. Knowles, J. Chem. Phys. 89, 5803 (1988).

${ }^{63}$ H.-J. Werner and P. J. Knowles, Chem. Phys. Lett. 145, 514 (1988).

${ }^{64}$ E. R. Davidson, J. Comput. Phys. 17, 87 (1975).

${ }^{65}$ A. Aguado and M. Paniagua, J. Chem. Phys. 96, 1265 (1992).

${ }^{66}$ A. Aguado, O. Roncero, C. Tablero, C. Sanz, and M. Paniagua, J. Chem. Phys. 112, 1240 (2000).

${ }^{67}$ See EPAPS Document No. E-JCPSA6-119-015343 for the four fit potential energy surfaces. A direct link to this document may be found in the online article's HTML reference section. The document may also be reached via the EPAPS homepage (http://www.aip.org/pubservs/ epaps.html) or from ftp.aip.org in the directory /epaps. See the EPAPS homepage for more information.

${ }^{68}$ R. N. Zare, Angular Momentum (Wiley, New York, 1988).

${ }^{69}$ R. Burcl, P. Piecuch, V. Spirko, and O. Bludský, Int. J. Quantum Chem. 80, 916 (2000).
${ }^{70}$ C. Lanczos, J. Res. Natl. Bur. Stand. 45, 255 (1950).

${ }^{71}$ J. K. Cullum and R. A. Willoughby, Lanczos Algorithms for Large Symmetric Eigenvalues Computations (Birkhäuser, Boston, 1985).

${ }^{72}$ C.-E. Fröberg, Numerical Mathematics: Theory and Computer Applications (Benjamin/Cummings, 1985).

${ }^{73}$ R. E. Wyatt, Adv. Chem. Phys. LXXIII, 231 (1989).

${ }^{74}$ M. S. Topaler, D. G. Truhlar, X. Y. Chang, P. Piecuch, and J. C. Polanyi, J. Chem. Phys. 108, 5378 (1998).

${ }^{75}$ C. Sanz, O. Roncero, C. Tablero, A. Aguado, and M. Paniagua, J. Chem. Phys. 114, 2182 (2001).

${ }^{76}$ R. Sadeghi and R. T. Skodje, J. Chem. Phys. 102, 193 (1995).

${ }^{77}$ C. L. Russell and D. E. Manolopoulos, Chem. Phys. Lett. 256, 465 (1996).

${ }^{78}$ A. Aguado, M. Paniagua, C. Sanz, and O. Roncero (unpublished).

${ }^{79}$ O. Roncero, N. Halberstadt, and J. A. Beswick, J. Chem. Phys. 104, 7554 (1996).

${ }^{80}$ H.-J. Werner, B. Follmeg, and M. H. Alexander, J. Chem. Phys. 89, 3139 (1988).

${ }^{81}$ D. Simah, B. Hartke, and H.-J. Werner, J. Chem. Phys. 111, 4523 (1999).

${ }^{82}$ T. E. Sharp, Atomic Data 2, 119 (1971).

${ }^{83}$ M. M. L. Chen and H. F. Schaefer III, J. Chem. Phys. 72, 4376 (1980).

${ }^{84}$ S. Carter and J. N. Murrell, Mol. Phys. 41, 567 (1980). 